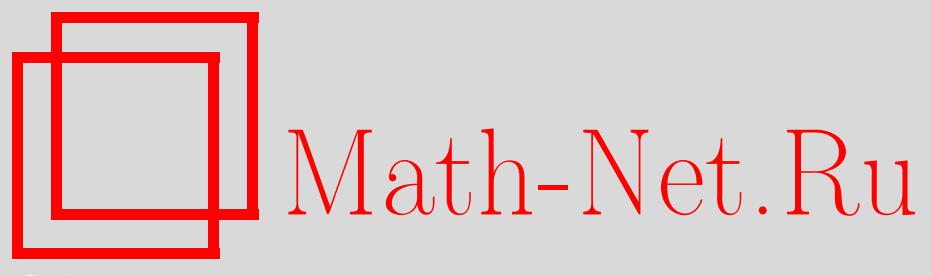

А. И. Буфетов, Эргодическое разложение для мер, квазиинвариантных относительно борелевских действий индуктивно компактных групп, Матем. сб., 2014, том 205, номер 2, 39-70

DOI: https://doi.org/10.4213/sm8202

Использование Общероссийского математического портала Math-Net.Ru подразумевает, что вы прочитали и согласны с пользовательским соглашением http://www . mathnet.ru/rus/agreement

Параметры загрузки:

IP: 34.239 .49 .27

26 апреля 2023 г., 16:31:41

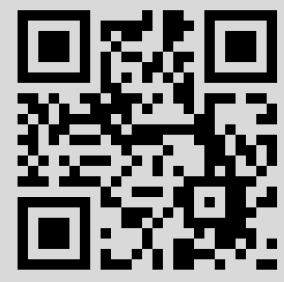




\title{
А. И. Буфетов \\ Эргодическое разложение для мер, квазиинвариантных относительно борелевских действий индуктивно компактных групп
}

\begin{abstract}
Цель настоящей работы - доказать теоремы об эргодическом разложении для вероятностных мер, квазиинвариантных относительно борелевских действий индуктивно компактных групп, и для $\sigma$-конечных инвариантных мер. Для бесконечных мер эргодическое разложение не единственно, но класс разлагаемой меры на пространстве проективных мер определен исходной инвариантной мерой однозначно.

Библиография: 21 название.
\end{abstract}

Ключевые слова: эргодическое разложение, бесконечномерные группы, квазиинвариантная мера, бесконечномерная унитарная группа, измеримое разбиение.

DOI: $10.4213 / \mathrm{sm} 8202$

\section{§1. Введение}

1.1. Сводка основных результатов. Первый результат этой работы утверждает существование и единственность эргодического разложения для вероятностных мер, квазиинвариантных относительно борелевских действий индуктивно компактных групп (теорема 1). Сначала мы показываем в предложении 2, что для действий индуктивно компактных групп эргодичность квазиинвариантной меры эквивалентна ее неразложимости; как показывает пример Колмогорова [1], эта эквивалентность не имеет места для сохраняющих меру действий общих групп. Тогда эргодическое разложение строится при дополнительном предположении, что коцикл Радона-Никодима меры непрерывен при ограничении на каждую орбиту группы (условие послойной непрерывности). Это условие ограничительно только для действий несчетных групп. Доказательство теоремы 1 основано на методе Рохлина построения эргодических разложений.

Работа поддержана в рамках проекта A*MIDEX (№ ANR-11-IDEX-0001-02), финансируемого французской государственной программой "Investissements d'Avenir" и французским национальным исследовательским агентством (ANR). Также работа частично поддержана исследовательской стипендией Альфреда П. Слоана, некоммерческим фондом "Династия", грантом Саймонса Независимого Московского университета, программой Президента РФ поддержки молодых кандидатов наук (грант № MK-6734.2012.1), программой Президента РФ поддержки молодых докторов наук (грант № МД-2859.2014.1), программой "Динамические системы и математическая теория управления" Президиума РАН, грантом PФФИ-CNRS 10-01-93115-NTsNIL и Российским фондом фундаментальных исследований (гранты №№ 11-01-00654, 12-01-31284, 12-01-33020 и 13-01-12449). 
Затем теорема 1 применяется к $\sigma$-конечным инвариантным мерам. В этом случае эргодическое разложение не единственно. Однако класс разложимой меры на пространстве проективных мер однозначно определяется исходной инвариантной мерой (теорема 2). В статьях [2] и [3] результаты настоящей работы применены к эргодическому разложению бесконечных мер Пикрелла, введенных Пикреллом [4], Бородиным и Ольшанским [5], на пространстве бесконечных комплексных матриц.

Для полноты изложения мы включили пример Колмогорова действия группы, допускающего разложимые эргодические меры.

1.2. Исторические комментарии. Идея эргодического разложения мер, вероятно, восходит к работе фон Неймана [6], см. также работу Крылова и Боголюбова [7]. Среди ранних работ также следует отметить замечательную статью Боголюбова [8]. Новый подход к проблеме эргодического разложения был предложен Рохлиным [9] и основывался на его теории измеримых разложений [10]. Подход Рохлина играет ключевую роль в этой статье.

Для действий группы $\mathbb{Z}$ с квазиинвариантной мерой теорема об эргодическом разложении была получена Кифером и Пироговым [11] с помощью метода Рохлина [9]. Более общие группы преобразований были рассмотрены Варадараяном [12], который, в частности, получил важный результат о реализации для борелевских действий локально компактных групп.

Для действий локально компактных групп общая теорема об эргодическом разложении принадлежит Грешонигу и Шмидту [13]. Грешониг и Шмидт предложили два подхода: в первом они следовали Рохлину и показали, что сигмаалгебра инвариантных множеств является "достаточной” для множества вероятностных мер с данным коциклом Радона-Никодима, второй подход основывался на теореме Шоке (см., например, [14]). Для того чтобы применить теорему Шоке, Грешониг и Шмидт использовали теорему Варадараяна [12], утверждающую, что каждое борелевское действие локально компактной группы допускает непрерывную реализацию. Не ясно, имеет ли место аналогичный результат для индуктивно компактных групп, и поэтому нет уверенности, что подход теоремы Шоке может быть непосредственно применен к действиям индуктивно компактных групп.

Для естественного действия бесконечной унитарной группы на пространстве бесконечных эрмитовых матриц эргодическое разложение инвариантных вероятностных мер было построено Бородиным и Ольшанским [5]. Бородин и Ольшанский [5] опирались на теорему Шоке, которая, однако, не могла быть непосредственно применена, поскольку пространство бесконечных эрмитовых матриц не компактно. Бородин и Ольшанский вложили пространство вероятностных мер на пространстве бесконечных эрмитовых матриц в большее выпуклое компактное метризуемое множество, к которому теорема Шоке применима. Подход Рохлина к проблеме эргодического разложения не требует ни непрерывности, ни компактности, и результаты этой статьи применимы ко всем 
борелевским действиям индуктивно компактных групп. Теорема о мартингальной сходимости используется вместо эргодической теоремы, на которую опираются аргументы Рохлина; идея использования мартингальной сходимости для изучения мер, инвариантных относительно действий индуктивно компактных групп, восходит к заметке Вершика [15].

\section{3. Измеримые действия топологических групп на борелевских пространствах.}

1.3.1. Стандартные борелевские пространства. Пусть $X$ - произвольное множество и $\mathscr{B}$ - сигма-алгебра на $X$. Пара $(X, \mathscr{B})$ называется стандартным борелевским пространством, если существует биекция между $X$ и единичным интервалом, которая переводит $\mathscr{B}$ в сигма-алгебру борелевских множеств. Будем называть $\mathscr{B}$ борелевской сигма-алгеброй, а меры, определенные на $\mathscr{B},-$ борелевскими мерами.

Пусть $\mathfrak{M}(X)$ - пространство борелевских вероятностных мер на $X$. Естественная сигма-алгебра $\mathscr{B}(\mathfrak{M}(X))$ на пространстве $\mathfrak{M}(X)$ определена следующим образом. Пусть $A \in X-$ борелевское подмножество, $\alpha \in \mathbb{R}$ и

$$
M_{A, \alpha}=\{\nu \in \mathfrak{M}(X): \nu(A)>\alpha\}
$$

Тогда сигма-алгебра $\mathscr{B}(\mathfrak{M}(X))$ - это минимальная сигма-алгебра, содержащая все множества $M_{A, \alpha}, A \in \mathfrak{B}(X), \alpha \in \mathbb{R}$. Ясно, что если $(X, \mathscr{B})$ - стандартное борелевское пространство, то $(\mathfrak{M}(X), \mathscr{B}(\mathfrak{M}(X))$ также является стандартным борелевским пространством. В самом деле, пространство вероятностных мер на единичном интервале является компактным метрическим пространством и, следовательно, стандартным борелевским пространством.

Борелевская мера $\nu$ на стандартном борелевском пространстве $(X, \mathscr{B})$ называется $\sigma$-конечной, если существует счетное семейство таких непересекающихся борелевских подмножеств

$$
X_{1}, X_{2}, \ldots, X_{n}, \ldots
$$

множества $X$, что

$$
X=\bigcup_{n=1}^{\infty} X_{n}
$$

и $\nu\left(X_{n}\right)<+\infty$ для любого $n \in \mathbb{N}$. Обозначим через $\mathfrak{M}^{\infty}(X)$ пространство всех $\sigma$-конечных борелевских мер на $X$ (отметим, что в нашей терминологии конечные меры также являются $\sigma$-конечными). Пространство $\mathfrak{M}^{\infty}(X)$ допускает естественную борелевскую структуру: борелевская сигма-алгебра порождается множествами вида

$$
\left\{\nu \in \mathfrak{M}^{\infty}(X): \alpha<\nu(A)<\beta\right\}
$$

где $\alpha, \beta$ - вещественные числа и $A$ - борелевское подмножество в $X$. 
Если $\nu$ - борелевская мера на $X$ и $f \in L_{1}(X, \nu)$, то для краткости обозначим

$$
\nu(f)=\int_{X} f d \nu .
$$

Как обычно, под классом мы подразумеваем семейство всех $\sigma$-конечных борелевских мер с той же сигма-алгеброй множеств меры нуль. Класс меры $\nu$ будем обозначать $[\nu]$. Будем писать $\nu_{1} \ll \nu_{2}$, если $\nu_{1}$ абсолютно непрерывна относительно $\nu_{2}$. Запись $\nu_{1} \perp \nu_{2}$ означает, как обычно, что меры $\nu_{1}$ и $\nu_{2}$ взаимно сингулярны.

1.3.2. Измеримые действия топологических групп. Пусть $G$ - топологическая группа, снабженная борелевской сигма-алгеброй, и, как и ранее, $(X, \mathscr{B})-$ стандартное борелевское пространство. Предположим, что группа $G$ действует на $X$, и для $g \in G$ пусть $T_{g}$ - соответствующее преобразование. Будем называть действие измеримым (или борелевским), если отображение

$$
\mathfrak{T}: G \times X \rightarrow X, \quad \mathfrak{T}(g, x)=T_{g} x,
$$

измеримо по Борелю. Группа $G$ действует на $\mathfrak{M}(X)$. Нам будет удобно рассматривать правое действие и ввести для $g \in G$ меру

$$
\nu \circ T_{g}(A)=\nu\left(T_{g} A\right)
$$

Получившееся правое действие, конечно, является борелевским.

1.3.3. Индуктивно компактные группы. Пусть

$$
K(1) \subset K(2) \subset \cdots \subset K(n) \subset \cdots
$$

- возрастающая цепочка метризуемых компактных групп, и пусть

$$
G=\bigcup_{n=1}^{\infty} K(n) .
$$

Тогда группа $G$ называется индуктивно компактной. Индуктивно компактная группа $G$ наделяется естественной топологией индуктивного предела, при которой функция на $G$ непрерывна тогда и только тогда, когда ее ограничение на каждую подгруппу $K(n)$ непрерывно. Борелевская сигма-алгебра на $G$ порождена борелевскими сигма-алгебрами на $K(n), n \in \mathbb{N}$.

Естественным примером является бесконечная унитарная группа

$$
U(\infty)=\bigcup_{n=1}^{\infty} U(n) .
$$

Индуктивный предел рассматривается относительно естественных включений. Группа $U(\infty)$, конечно, не является локально компактной (см., например, [16]). 


\section{4. Коциклы и меры.}

1.4.1. Измеримые коциклы. В этой работе измеримый коцикл над измеримым действием $\mathfrak{T}$ топологической группы $G$ всегда означает положительный вещественнозначный мультипликативный коцикл, т.е. измеримое отображение

$$
\rho: G \times X \rightarrow \mathbb{R}_{>0}
$$

удовлетворяющее условию коцикла

$$
\rho(g h, x)=\rho\left(g, T_{h} x\right) \cdot \rho(h, x),
$$

см., например, книгу К. Шмидта [17]. Пусть дан положительный вещественнозначный мультипликативный коцикл $\rho$ над измеримым действием т топологической группы $G$. Введем пространство $\mathfrak{M}(\mathfrak{T}, \rho) \subset \mathfrak{M}(X)$ измеримых по Борелю мер с коциклом Радона-Никодима $\rho$ по отношению к действию т:

$$
\begin{gathered}
\mathfrak{M}(\mathfrak{T}, \rho)=\left\{\nu \in \mathfrak{M}(X): \frac{d \nu \circ T_{g}}{d \nu}(x)=\rho(g, x) \text { для всех } g \in G\right. \\
\text { и } \nu \text {-почти всех } x \in X\} .
\end{gathered}
$$

Заметим, что для данной вероятностной меры $\nu$, квазиинвариантной относительно действия $\mathfrak{T}$, ее коцикл Радона-Никодима не единственен, но определен однозначно почти везде: если есть два коцикла Радона-Никодима $\rho_{1}, \rho_{2}$, которые соответствуют одной и той же мере $\nu$, то для любого $g \in G$ равенство

$$
\rho_{1}(g, x)=\rho_{2}(g, x)
$$

справедливо для $\nu$-почти всех $x \in X$.

Однако пространство $\mathfrak{M}(\mathfrak{T}, \rho)$ является выпуклым конусом. Действительно, оно замкнуто относительно умножения на положительные константы, и если

$$
\nu_{i} \circ T_{g}(A)=\int_{A} \rho(g, x) d \nu_{i}, \quad i=1,2,
$$

то также

$$
\left(\nu_{1}+\nu_{2}\right) \circ T_{g}(A)=\int_{A} \rho(g, x) d\left(\nu_{1}+\nu_{2}\right) .
$$

1.4.2. Неразложимость и эргодичность. Пусть, как и ранее, $\rho$ - положительный вещественнозначный мультипликативный измеримый коцикл над измеримым действием $\mathfrak{T}$ группы $G$ на стандартном борелевском пространстве $(X, \mathscr{B})$.

Мера $\nu \in \mathfrak{M}(\mathfrak{T}, \rho)$ называется неразложимой в $\mathfrak{M}(\mathfrak{T}, \rho)$, если из равенства $\nu=\alpha \nu_{1}+(1-\alpha) \nu_{2}$, где $\alpha \in(0,1), \nu_{1}, \nu_{2} \in \mathfrak{M}(\mathfrak{T}, \rho)$, следует $\nu=\nu_{1}=\nu_{2}$.

Напомним, что борелевское множество $A$ называется почти инвариантнъм относительно борелевской меры $\nu$, если для каждого $g \in G$ выполнено $\nu\left(A \triangle T_{g} A\right)=0$. Неразложимость может быть эквивалентно переформулирована следующим образом (см. [8], [1]). 
ПрЕДлОЖЕНИЕ 1. Борелевская вероятностная мера $\nu \in \mathfrak{M}(\mathfrak{T}, \rho)$ является неразложимой в $\mathfrak{M}(\mathfrak{T}, \rho)$ тогда и только тогда, когда любое борелевское множество A, почти инвариантное относительно действия $\mathfrak{T}$ по отношению $\kappa$ мере $\nu$, удовлетворяет либо условию $\nu(A)=0$, либо условию $\nu(X \backslash A)=0$.

ЗАмечАниЕ. Отметим, что в этом предложении нет ограничений на действующую группу.

Для полноты изложения доказательство предложения 1 приведено в $\S 5$.

Мера $\nu \in \mathfrak{M}(\mathfrak{T}, \rho)$ называется эргодической, если для каждого $G$-инвариантного борелевского множества $A$ либо $\nu(A)=0$, либо $\nu(X \backslash A)=0$. Множество всех эргодических мер с коциклом Радона-Никодима $\rho$ обозначается $\mathfrak{M}_{\operatorname{erg}}(\mathfrak{T}, \rho)$.

Неразложимые меры заведомо являются эргодическими. Для действий общих групп эргодические вероятностные меры могут не быть неразложимыми: как показал Колмогоров, эти два понятия различны для естественного действия группы всех биекций $\mathbb{Z}$ на пространстве бесконечных в обе стороны бинарных последовательностей (для полноты изложения мы напомним пример Колмогорова в §5). Неформально, причина в том, что действия "больших" групп могут иметь “слишком мало” орбит (в примере Колмогорова - счетное множество), и, следовательно, выпуклая комбинация различных эргодических мер также может быть эргодической.

Тем не менее для действий индуктивно компактных групп эти понятия совпадают.

ПреДЛОЖЕНИЕ 2. Пустъ $\mathfrak{T}$ - измеримое действие индуктивно компактной группь $G$ на стандартном борелевском пространстве $(X, \mathscr{B})$, и пусть $\rho$ - положительный измеримый мультипликативный коиикл над Т. Если мера $\nu \in \mathfrak{M}(\mathfrak{T}, \rho)$ является эргодической, то д разложима в $\mathfrak{M}(\mathfrak{T}, \rho)$.

Предложение 2 будет доказано в $§ 2$.

\section{5. Эргодическое разложение квазиинвариантных вероятностных мep.}

1.5.1. Послойно непрерывные коциклы. Для того чтобы сформулировать теорему об эргодическом разложении для квазиинвариантных мер, нам надо наложить дополнительные условия на коцикл Радона-Никодима $\rho$.

Пусть $\mathfrak{T}$ - измеримое действие топологической группы $G$ на стандартном борелевском пространстве $(X, \mathscr{B})$.

ОПреДЕлЕНиЕ. Положительный вещественнозначный измеримый коцикл $\rho: G \times X \rightarrow \mathbb{R}_{>0}$ над действием $\mathfrak{T}$ назовем послойно непрерьвным, если для любого $x \in X$ функция $\rho_{x}: G \rightarrow \mathbb{R}_{>0}$, заданная формулой $\rho_{x}(g)=\rho(g, x)$, непрерывна.

ЗАмЕчАниЕ. Если $G$ индуктивно компактна,

$$
G=\bigcup_{n=1}^{\infty} K(n), \quad K(n) \subset K(n+1),
$$


то по определению топологии индуктивного предела требование послойной непрерывности означает в точности, что для любого $n \in \mathbb{N}$ функция $\rho_{x}$, определенная выше, непрерывна после ограничения на $K(n)$.

Для общих действий топологических групп не ясно, является ли множество мер с данным коциклом Радона-Никодима борелевским. Это так, однако, для действий индуктивно компактных групп и послойно непрерывных коциклов.

ПРЕДЛОЖЕНИЕ 3. Пусть $\rho$ - послойно непрерывный коиикл над измеримым действием т локально компактной дважды счетной группы $G$ на стандартном борелевском пространстве $(X, \mathscr{B})$. Тогда множество $\mathfrak{M}(\mathfrak{T}, \rho)$ является борелевским подмножеством множества $\mathfrak{M}(X)$.

ДокАЗАтельство. Сначала заметим, что для любого фиксированного $g \in G$ множество

$$
\left\{\nu \in \mathfrak{M}(X): \frac{d \nu \circ T_{g}}{d \nu}=\rho(g, x) \quad \nu \text {-почти наверное }\right\}
$$

является борелевским по определению.

Напомним следующую теорему реализации Варадараяна (теорема 3.2 в [12]).

ТЕорема (Варадараян). Предположим, что локально компактная дважды счетная группа $K$ действует измеримо на стандартном борелевском пространстве $(X, \mathscr{B})$. Тогда существуют такие компактное метрическое пространство $Z$, непрерывное действие $K$ на $Z$ и $K$-инвариантное борелевское подмножество $Z^{\prime} \subset Z$, что ограниченное действие $K$ на $Z^{\prime}$ измеримо изоморфно действию $K$ на $(X, \mathscr{B})$.

В силу данной теоремы можно считать, что действие $G$ непрерывно. Выбрав счетную плотную подгруппу в $G$ и взяв предел, мы завершим доказательство предложения 3 .

Применяя предложение 3 к каждой из компактных подгрупп, получаем

СЛЕДСТВИЕ 1. Пусть $\rho$ - послойно непрерывный коиикл над измеримым действием T индуктивно компактной дважды счетной группь $G$ на стандартном борелевском пространстве $(X, \mathscr{B})$. Тогда множество $\mathfrak{M}(\mathfrak{T}, \rho)$ является борелевским подмножеством в $\mathfrak{M}(X)$.

ПрЕДЛОЖЕНИЕ 4. Пусть $\rho$ - послойно непрерывный коиикл над измеримым действием T индуктивно компактной группы $G$ на стандартном борелевском пространстве $(X, \mathscr{B})$. Тогда множество $\mathfrak{M}_{\operatorname{erg}}(\mathfrak{T}, \rho)$ является борелевским подмножеством в $\mathfrak{M}(X)$.

Это предложение будет доказано в 2 .

Вопрос. При каких условиях заключение теоремы реализации Варадараяна выполнено для борелевских действий индуктивно компактных групп? 
1.5.2. Интегралы над пространством мер. Пусть $\widetilde{\nu} \in \mathfrak{M}(\mathfrak{M}(X))$, или, другими словами, $\widetilde{\nu}-$ борелевская вероятностная мера на пространстве борелевских вероятностных мер на $X$. Введем меру $\nu \in \mathfrak{M}(X)$ формулой

$$
\nu=\int_{\mathfrak{M}(X)} \eta d \widetilde{\nu}(\eta) .
$$

Интеграл в правой части равенства (1) понимается в следующем слабом смысле. Для любого борелевского множества $A \subset X$ функция $\operatorname{int}_{A}: \mathfrak{M}(X) \rightarrow \mathbb{R}$, заданная формулой $\operatorname{int}_{A}(\eta)=\eta(A)$, очевидно, измерима по Борелю. Равенство (1) означает, что для любого борелевского множества $A \subset X$ мы имеем

$$
\nu(A)=\int_{\mathfrak{M}(X)} \eta(A) d \widetilde{\nu}(\eta) .
$$

1.5.3. Теорема об эргодическом разложении. Следующая формулировка теоремы об эргодическом разложении состоит из четырех утверждений: первые два устанавливают существование эргодического разложения, третье утверждает его единственность, а четвертое связывает абсолютную непрерывность квазиинвариантных мер с абсолютной непрерывностью их разложений.

Теорема 1. Пусть т - измеримое действие индуктивно компактной группы $G$ на стандартном борелевском пространстве $(X, \mathscr{B})$. Пусть $\rho$ послойно непрерывный положительный вещественнозначный мультипликативный коиикл над T. Тогда существуют такие борелевское подмножество $\widetilde{X} \subset X$ и сюргективное борелевское отображение

$$
\pi: \widetilde{X} \rightarrow \mathfrak{M}_{\operatorname{erg}}(\mathfrak{T}, \rho),
$$

что

(1) для любого $\eta \in \mathfrak{M}_{\operatorname{erg}}(\mathfrak{T}, \rho)$ имеем $\eta\left(\pi^{-1}(\eta)\right)=1$;

(2) для любого $\nu \in \mathfrak{M}(\mathfrak{T}, \rho)$

$$
\nu=\int_{\mathfrak{M}_{\operatorname{erg}}(\mathfrak{T}, \rho)} \eta d \bar{\nu}(\eta),
$$

где $\bar{\nu}=\pi_{*} \nu$. В частности, для любого $\nu \in \mathfrak{M}(\mathfrak{T}, \rho)$ имеем $\nu(\widetilde{X})=1$;

(3) соответствие $\nu \rightarrow \bar{\nu}$ является борелевским изоморфизмом между борелевскими пространствами $\mathfrak{M}(\mathfrak{T}, \rho)$ и $\mathfrak{M}\left(\mathfrak{M}_{\operatorname{erg}}(\mathfrak{T}, \rho)\right)$, и если $\nu \in \mathfrak{M}(\mathfrak{T}, \rho)$ и $\widetilde{\nu} \in \mathfrak{M}\left(\mathfrak{M}_{\operatorname{erg}}(\mathfrak{T}, \rho)\right)$ таковы, что выполнено

$$
\nu=\int_{\mathfrak{M}_{\operatorname{erg}}(\mathfrak{T}, \rho)} \eta d \widetilde{\nu}(\eta),
$$

mo $\widetilde{\nu}=\bar{\nu}$

(4) для любых $\nu_{1}, \nu_{2} \in \mathfrak{M}_{\operatorname{erg}}(\mathfrak{T}, \rho)$ имеем $\nu_{1} \ll \nu_{2}$ тогда и только тогда, когда $\bar{\nu}_{1} \ll \bar{\nu}_{2}, u \nu_{1} \perp \nu_{2}$ тогда и только тогда, когда $\bar{\nu}_{1} \perp \bar{\nu}_{2}$. 


\section{6. Эргодическое разложение бесконечных инвариантных мер.}

1.6.1. Редукция к эквивалентной конечной мере. Теперь мы применим полученные результаты к борелевским действиям, сохраняющим бесконечную меру. Пусть дано измеримое действие $\mathfrak{T}$ группы $G$. Обозначим через $\mathfrak{M}_{\text {inv }}^{\infty}(\mathfrak{T})$ подмножество $G$-инвариантных мер в $\mathfrak{M}^{\infty}$ и через $\mathfrak{M}_{\mathrm{erg}}^{\infty}(\mathfrak{T})$ подмножество $G$-инвариантных эргодических мер в $\mathfrak{M}^{\infty}$. Не ясно, являются ли множества $\mathfrak{M}_{\text {inv }}^{\infty}(\mathfrak{T})$ и $\mathfrak{M}_{\mathrm{erg}}^{\infty}(\mathfrak{T})$ борелевскими. Поэтому удобно рассматривать меньшие подмножества в $\mathfrak{M}^{\infty}$, а именно подмножества мер, которые сопоставляют конечный интеграл данной положительной измеримой функции.

Для упрощения обозначений, рассматривая фиксированное пространство $X$, мы опускаем его в обозначениях. Например, пишем $\mathfrak{M}$ вместо $\mathfrak{M}(X)$. Также для меры $\nu \in \mathfrak{M}^{\infty}$ и функции $f \in L_{1}(X, \nu)$ пишем

$$
\nu(f)=\int f d \nu .
$$

Для положительной измеримой функции $f$ на $X$ положим

$$
\mathfrak{M}_{f}^{\infty}=\left\{\nu \in \mathfrak{M}^{\infty}: f \in L_{1}(X, \nu)\right\}
$$

Определим отображение

$$
P_{f}: \mathfrak{M}_{f}^{\infty} \rightarrow \mathfrak{M}
$$

формулой

$$
P_{f}(\nu)=\frac{f \nu}{\nu(f)}
$$

Введем коцикл $\rho_{f}$ над действием $\mathfrak{T}$ формулой

$$
\rho_{f}(g, x)=\frac{f\left(T_{g} x\right)}{f(x)} .
$$

Мера $\nu \in \mathfrak{M}_{f}^{\infty}$ является $\mathfrak{T}$-инвариантной тогда и только тогда, когда

$$
P_{f}(\nu) \in \mathfrak{M}\left(\mathfrak{T}, \rho_{f}\right) .
$$

Обозначим

$$
\begin{gathered}
\mathfrak{M}_{f, \text { inv }}^{\infty}(\mathfrak{T})=\mathfrak{M}_{f}^{\infty} \cap \mathfrak{M}_{\text {inv }}^{\infty}(\mathfrak{T}), \quad \mathfrak{M}_{f, \text { erg }}^{\infty}(\mathfrak{T})=\mathfrak{M}_{f}^{\infty} \cap \mathfrak{M}_{\mathrm{erg}}^{\infty}(\mathfrak{T}), \\
\mathfrak{M}_{f, 1}^{\infty}=\left\{\nu \in \mathfrak{M}_{f}^{\infty}: \nu(f)=1\right\}, \\
\mathfrak{M}_{f, 1, \text { inv }}^{\infty}(\mathfrak{T})=\mathfrak{M}_{f, 1}^{\infty} \cap \mathfrak{M}_{\text {inv }}^{\infty}(\mathfrak{T}), \quad \mathfrak{M}_{f, 1, \operatorname{erg}}^{\infty}(\mathfrak{T})=\mathfrak{M}_{f, 1}^{\infty} \cap \mathfrak{M}_{\mathrm{erg}}^{\infty}(\mathfrak{T}) .
\end{gathered}
$$

Множество $\mathfrak{M}_{f, 1}^{\infty}$ является борелевским по определению. Отображение $P_{f}$ определяет борелевский изоморфизм борелевских пространств $\mathfrak{M}_{f, 1}^{\infty}$ и $\mathfrak{M}$, следовательно, последнее - стандартное борелевское пространство. Далее, мы имеем

$$
P_{f}\left(\mathfrak{M}_{f, 1, \mathrm{inv}}^{\infty}\right)=\mathfrak{M}\left(\mathfrak{T}, \rho_{f}\right), \quad P_{f}\left(\mathfrak{M}_{f, 1, \mathrm{erg}}^{\infty}\right)=\mathfrak{M}_{\operatorname{erg}}\left(\mathfrak{T}, \rho_{f}\right)
$$


Для того чтобы примерить теорему 1 к $\mathfrak{M}\left(\mathfrak{T}, \rho_{f}\right)$, нам нужно дополнительное ограничение на функцию $f$.

ОПРЕдЕЛЕНиЕ. Будем говорить, что измеримая по Борелю функция

$$
f: X \rightarrow \mathbb{R}
$$

послойно непрерывна, если для любого $x \in X$ функция $f\left(T_{g} x\right)$ непрерывна B $g \in G$.

В частности, если $X$ - метрическое пространство и действие $\mathfrak{T}$ само непрерывно, то любая непрерывная функция a fortiori послойно непрерывна. Чтобы получить непрерывные интегрируемые функции, используется следующее простое утверждение.

ПреДЛОЖЕНИЕ 5. Пусть $X$ - метрическое пространство и $\nu$ - -конечная борелевская мера на $X$, сопоставляющая конечный вес каждому шару. Тогда пространство $L_{1}(X, \nu)$ содержит положительные непрерывные функции.

ДокАзАтельство. Пусть $d$ - метрика на $X$. Возьмем $x_{0} \in X$. Пусть $\psi: \mathbb{R}_{+} \rightarrow \mathbb{R}_{>0}-$ положительная ограниченная непрерывная функция. Положим $f(x)=\psi\left(d\left(x, x_{0}\right)\right)$. Вес каждого шара конечен, поэтому если функция $\psi$ достаточно быстро убывает на бесконечности, то $f \in L_{1}(X, \nu)$.

Если функция $f$ послойно непрерывна, то коцикл $\rho_{f}$, заданный формулой

$$
\rho_{f}(g, x)=\frac{f\left(T_{g} x\right)}{f(x)},
$$

также является послойно непрерывным. Следовательно, множества $M_{f, 1, \mathrm{inv}}^{\infty}$ и $M_{f, 1, \mathrm{erg}}^{\infty}$ являются борелевскими подмножествами в $\mathfrak{M}^{\infty}$ и такими же являются $\mathfrak{M}_{f, \text { inv }}^{\infty}$ и $\mathfrak{M}_{f, \text { erg }}^{\infty}$.

Без ограничения общности можно считать, что $\nu(f)=1$, и рассмотреть эргодическое разложение

$$
f \nu=\int_{\mathfrak{M}_{\operatorname{erg}}\left(\mathfrak{T}, \rho_{f}\right)} \eta d \check{\nu}(\eta)
$$

меры $f \nu$ в $\mathfrak{M}\left(\mathfrak{T}, \rho_{f}\right)$. Разделив на $f$, получим эргодическое разложение

$$
\nu=\int_{\mathfrak{M}_{f, 1, \operatorname{erg}}^{\infty}} \eta d \widetilde{\nu}(\eta)
$$

исходной меры $\nu$; заметим, что по построению соответствие $\nu \rightarrow \widetilde{\nu}$ является биекцией. 
Из теоремы 1 получаем

СледСтвиЕ 2. Пусть $\mathfrak{T}$ - измеримое действие индуктивно компактной группы $G$ на стандартном борелевском пространстве $(X, \mathscr{B}), f: X \rightarrow \mathbb{R}_{>0}-$ измеримая положительная послойно непрерывная функция. Тогда:

(1) множества $\mathfrak{M}_{f, 1, \mathrm{inv}}^{\infty}(\mathfrak{T})$ u $\mathfrak{M}_{f, 1, \mathrm{erg}}^{\infty}(\mathfrak{T})$ - борелевские подмножества в $\mathfrak{M}^{\infty}(X)$;

(2) каждая мера $\eta \in \mathfrak{M}_{f, 1, \operatorname{erg}}^{\infty}(\mathfrak{T})$ неразложима в $\mathfrak{M}_{f, 1, \mathrm{inv}}^{\infty}(\mathfrak{T})$;

(3) для любой $\nu \in \mathfrak{M}_{f, 1, \text { inv }}^{\infty}(\mathfrak{T})$ существует единственная борелевская вероятностная мера $\bar{\nu}$ на $\mathfrak{M}_{f, 1, \mathrm{erg}}^{\infty}(\mathfrak{T})$ такая, что

$$
\nu=\int_{\mathfrak{M}_{f, 1, \operatorname{erg}}^{\infty}(\mathfrak{T})} \eta d \bar{\nu}(\eta) .
$$

Биективное соответствие $\nu \rightarrow \bar{\nu}$ является борелевским изоморфизмом борелевских пространств $\mathfrak{M}_{f, 1, \mathrm{inv}}^{\infty}(\mathfrak{T})$ u $\mathfrak{M}\left(\mathfrak{M}_{f, 1, \mathrm{erg}}^{\infty}(\mathfrak{T})\right)$.

Из следствия 2 непосредственно вытекает

СледСтвиЕ 3. Пусть Т - измеримое действие индуктивно компактной группы $G$ на стандартном борелевском пространстве $(X, \mathscr{B})$ и $\nu$ - такая $\sigma$ конечная T-инвариантная борелевская мера на $X$, что пространство $L_{1}(X, \nu)$ содержит положительную измеримую по Борелю послойно непрерывную функиию. Тогда мера $\nu$ допускает эргодическое разложение.

Действительно, эргодическое разложение получается, если взять положительную измеримую по Борелю послойно непрерывную функцию $f \in L_{1}(X, \nu)$ и разделить на $f$ разложение (6) меры $f \nu$. Такое эргодическое разложение, конечно, не единственно и зависит от выбора положительной измеримой по Борелю послойно непрерывной интегрируемой функции.

Удобно разрешить более общие эргодические разложения бесконечных мер. Пусть дана мера $\nu \in \mathfrak{M}^{\infty}(X)$ и $\sigma$-конечная борелевская мера $\bar{\nu}$ на $\mathfrak{M}^{\infty}(X)$. Равенство

$$
\nu=\int_{\mathfrak{M}^{\infty}(X)} \eta d \bar{\nu}(\eta)
$$

всегда будет пониматься так же, как и выше, в следующем слабом смысле. Пусть дано борелевское множество $A$. Как и ранее, рассмотрим функцию

$$
\operatorname{int}_{A}: \mathfrak{M}^{\infty} \rightarrow \mathbb{R}_{\geqslant 0} \cup\{\infty\}
$$

определенную как

$$
\operatorname{int}_{A}(\eta)=\eta(A)
$$

Равенство (7) означает, что для любого борелевского множества $A$, удовлетворяющего условию $\nu(A)<+\infty$, имеем $\operatorname{int}_{A} \in L_{1}\left(\mathfrak{M}^{\infty}(X), \bar{\nu}\right)$ и

$$
\nu(A)=\int_{\mathfrak{M}^{\infty}(X)} \eta(A) d \bar{\nu}(\eta) .
$$


Для меры $\nu$, инвариантной относительно действия $\mathfrak{T}$, разложение

$$
\nu=\int_{\mathfrak{M} \infty(X)} \eta d \bar{\nu}(\eta)
$$

назовем эргодическим разложением $\nu$, если $\bar{\nu}$ является $\sigma$-конечной мерой на $\mathfrak{M}^{\infty}(X)$ и $\bar{\nu}$-почти все меры $\eta \in \mathfrak{M}^{\infty}(X)$ инвариантны и эргодичны по отношению к действию $\mathfrak{T}$. Такое разложение, конечно, далеко не единственно: действительно, если

$$
\varphi: \mathfrak{M}^{\infty}(X) \rightarrow \mathbb{R}
$$

- такая измеримая по Борелю функция, что $\varphi(\eta)>0$ для $\bar{\nu}$-почти всех $\eta$, то новое разложение получается, если написать

$$
\nu=\int_{\mathfrak{M}^{\infty}(X)} \frac{\eta}{\varphi(\eta)} d(\varphi(\eta) \bar{\nu}(\eta)) .
$$

1.6.2. Проективные меры и допустимость. Как и выше, мы считаем пространство $X$ фиксированным и опускаем его в обозначениях. Введем проективное пространство $\mathbb{P} \mathfrak{M}^{\infty}$ - факторпространство пространства $\mathfrak{M}^{\infty}$ по отношению проективной эквивалентности $\sim$, определенному следующим образом:

$$
\nu_{1} \sim \nu_{2}, \quad \text { если } \nu_{1}=\lambda \nu_{2} \text { для некоторого } \lambda>0 \text {. }
$$

Пусть

$$
\mathbf{p}: \mathfrak{M}^{\infty} \rightarrow \mathbb{P M}^{\infty}
$$

- естественная проекция. Элементы из $\mathbb{P M}^{\infty}$ будем называть проективными мерами; конечность, инвариантность, квазиинвариантность и эргодичность проективных мер определяются очевидным способом. Обозначим

$$
\mathbb{P} \mathfrak{M}_{\text {inv }}^{\infty}(\mathfrak{T})=\mathbf{p}\left(\mathfrak{M}_{\text {inv }}^{\infty}(\mathfrak{T})\right), \quad \mathbb{P} \mathfrak{M}_{\text {erg }}^{\infty}(\mathfrak{T})=\mathbf{p}\left(\mathfrak{M}_{\text {erg }}^{\infty}(\mathfrak{T})\right)
$$

Борелевская структура на пространстве $\mathbb{P M}^{\infty}$ определяется естественным образом: множество $A \subset \mathbb{P} \mathfrak{M}^{\infty}$ является борелевским, если его прообраз $\mathbf{p}^{-1}(A)$ борелевский.

ОПРЕДЕлЕниЕ. Мера $\bar{\nu} \in \mathfrak{M}^{\infty}\left(\mathfrak{M}^{\infty}\right)$ называется допустимой, если проективное отображение $\mathbf{p} \bar{\nu}$-почти наверное биективно.

$\mathrm{K}$ примеру, любая мера с носителем на множестве $\mathfrak{M}^{\infty}(\mathfrak{M})$ или, для положительной измеримой $f$, на множестве $\mathfrak{M}^{\infty}\left(\mathfrak{M}_{f, 1}^{\infty}\right)$ автоматически является допустимой.

Если мера $\bar{\nu}$ в эргодическом разложении (8) является допустимой, то эргодическое разложение также называется допустимым.

Следующая теорема показывает, что для данной инвариантной $\sigma$-конечной меры $\nu$ класс меры $\mathbf{p}_{*} \bar{\nu}$ один и тот же для всех допустимых эргодических разложений (8). 
ТЕОРема 2. Пусть $\mathfrak{T}$ - измеримое действие индуктивно компактной группы $G$ на стандартном борелевском пространстве $(X, \mathscr{B})$ и $\nu$ - такая $\sigma$ конечная T-инвариантная борелевская мера на $X$, что пространство $L_{1}(X, \nu)$ содержит положительную измеримую по Борелю послойно непрерывную функиию. Тогда существует класс $\operatorname{PCL}(\nu)$ на $\mathbb{P M}^{\infty}$ со следующими свойствами.

(1) Для любой $\widetilde{\nu} \in \mathrm{PCL}(\nu)$ имеем $\widetilde{\nu}\left(\mathbb{P} \mathfrak{M}^{\infty} \backslash \mathbb{P M}_{\mathrm{erg}}^{\infty}(\mathfrak{T})\right)=0$.

(2) Для любого допустимого эргодического разложения

$$
\nu=\int_{\mathfrak{M}^{\infty}} \eta d \bar{\nu}(\eta)
$$

меры $\nu$ выполнено $\mathbf{p}_{*} \bar{\nu} \in \operatorname{PCL}(\nu)$.

(3) Обратно, для любой $\sigma$-конечной борелевской меры $\widetilde{\nu} \in \operatorname{PCL}(\nu)$ существует единственная допустимая $\sigma$-конечная борелевская мера $\bar{\nu}$ на $\mathfrak{M}^{\infty}(X)$ такая, что $\mathbf{p}_{*} \bar{\nu}=\widetilde{\nu} u$

$$
\nu=\int_{\mathfrak{M} \infty(X)} \eta d \bar{\nu}(\eta) .
$$

(4) Пусть $\nu_{1} и \nu_{2}$ - две T-инвариантные $\sigma$-конечные борелевские меры, каждая из которых допускает положительную послойно непрерывную интегрируемую функиию. Тогда $\nu_{1} \ll \nu_{2}$, если и только если $\operatorname{PCL}\left(\nu_{1}\right) \ll \operatorname{PCL}\left(\nu_{2}\right), u$ $\nu_{1} \perp \nu_{2}$, если и только если $\operatorname{PCL}\left(\nu_{1}\right) \perp \operatorname{PCL}\left(\nu_{2}\right)$. В частности, $\operatorname{PCL}\left(\nu_{1}\right)=$ $\operatorname{PCL}\left(\nu_{2}\right)$, если и только если $\left[\nu_{1}\right]=\left[\nu_{2}\right]$.

1.6.3. Бесконечные меры, все эргодические компоненты которых конечны. Рассмотрим множество $\mathbb{P} \mathfrak{M}$ конечных проективных мер. Пусть $\nu$ - такая $\sigma$ конечная инвариантная мера, что $\mathrm{PCL}(\nu)$ сосредоточена на $\mathbb{P} \mathfrak{M}$. В этом случае возьмем произвольное эргодическое разложение

$$
\nu=\int_{\mathfrak{M} \infty} \eta d \widetilde{\nu}(\eta)
$$

и изменим его, записав

$$
\nu=\int_{\mathfrak{M} \infty} \frac{\eta}{\eta(1)} \eta(1) d \widetilde{\nu}(\eta) .
$$

Таким образом, мы получим эргодическое разложение

$$
\nu=\int_{\mathfrak{M}_{\operatorname{erg}}(\mathfrak{T})} \eta d \bar{\nu}(\eta),
$$

где мера $\bar{\nu}$ сосредоточена на $\mathfrak{M}_{\operatorname{erg}}(\mathfrak{T})$ и однозначно определена мерой $\nu$.

Автор глубоко признателен Григорию Ольшанскому, поставившему перед ним эту задачу. Автор благодарен Клаусу Шмидту за объяснение конструкции из $\S 5$ работы [13] и за многочисленные полезные обсуждения. Автор благодарит Ива Кудена за объяснения теории Суслина. Автор благодарит Вадима Каймановича и Севака Мкртчяна за полезные обсуждения. Автор благодарит 
рецензента за многочисленные полезные предложения по улучшению изложения. Автор благодарит Никиту Козина за набор части этого текста. Часть этой работы была выполнена во время пребывания в Институте Миттаг-Леффлера в Дюрсхольме, в институте Эрвина Шредингера в Вене и в институте Макса Планка в Бонне; автор искренне признателен этим организациям за гостеприимство.

\section{§ 2. Операторы усреднения}

2.1. Усреднение по орбитам компактных групп. Пусть $K$ - компактная группа, снабженная мерой Хаара $\mu_{K}$ и $\mathfrak{T}_{K}$ - измеримое действие группы $K$ на стандартном борелевском пространстве $(X, \mathscr{B})$. Пусть $\rho$ - положительный мультипликативный вещественнозначный коцикл над действием $\mathfrak{T}_{K}$. Пусть $\mathbb{B}(X)$ - пространство ограниченных измеримых функций на $X$, снабженное равномерной метрикой Чебышёва. Введем оператор $\mathscr{A}_{K}^{\rho}: \mathbb{B}(X) \rightarrow \mathbb{B}(X)$ по формуле

$$
\left(\mathscr{A}_{K}^{\rho} f\right)(x)= \begin{cases}\frac{\int_{K} f\left(T_{k} x\right) \rho(k, x) d \mu_{K}(k)}{\int_{K} \rho(k, x) d \mu_{K}(k)}, & \int_{K} \rho(k, x) d \mu_{K}(k)<+\infty \\ 0, & \int_{K} \rho(k, x) d \mu_{K}(k)=+\infty .\end{cases}
$$

Ясно, что $\mathscr{A}_{K}^{\rho}$ - положительное стягивание на пространстве $\mathbb{B}(X)$.

Пусть $\mathscr{I}_{K}$ - сигма-алгебра $K$-инвариантных подмножеств множества $X$, и для данной меры $\nu$ пусть $\mathscr{I}_{K}^{\nu}-$ пополнение $\mathscr{I}_{K}$ относительно $\nu$.

Как и ранее, $\mathfrak{M}\left(\mathfrak{T}_{K}, \rho\right)$ обозначает пространство борелевских вероятностных мер на $X$ с коциклом Радона-Никодима $\rho$ по отношению к действию $\mathfrak{T}_{K}$.

Лемма 1. Для любой $\nu \in \mathfrak{M}\left(\mathfrak{T}_{K}, \rho\right)$ и любой $f \in L_{1}(X, \nu)$ оба интеграла

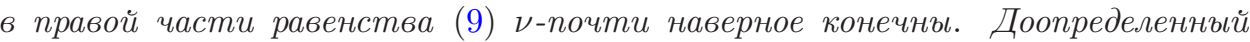
оператор $\mathscr{A}_{K}^{\rho}$ является положительным стягиванием на $L_{1}(X, \nu)$, и мы имеем $\nu$-почти наверное выполненное равенство

$$
\mathscr{A}_{K}^{\rho} f=\mathrm{E}\left(f \mid \mathscr{I}_{K}^{\nu}\right) .
$$

ЗАмечаниЕ. Левая часть равенства (10) зависит не от меры $\nu$, но только от коцикла $\rho$. Это простое наблюдение будет важно в дальнейшем. В терминологии Грешонига и Шмидта [13] лемма 1 утверждает, что сигма-алгебра $K$-инвариантных множеств достаточна для $\mathfrak{M}\left(\mathfrak{T}_{K}, \rho\right)$. Используя обратную теорему о мартингальной сходимости, мы покажем, что для действий индуктивно компактных групп сигма-алгебра инвариантных борелевских множеств достаточна для пространства вероятностных мер с фиксированным послойно непрерывным коциклом Радона-Никодима, а также что сигма-алгебра инвариантных борелевских множеств счетно порождена по модулю универсальных нуль-множеств, т.е. множеств меры нуль относительно всех вероятностных мер с фиксированным послойно непрерывным коциклом Радона-Никодима. 
ДокАЗАТЕЛЬСтво лЕммы 1 . Пусть $\rho_{x}: K \rightarrow \mathbb{R}$ задано формулой

$$
\rho_{x}(k)=\rho(k, x) .
$$

Из теоремы Фубини немедленно следует, что для $\nu$-почти всех $x \in X$ выполнено $\rho_{x} \in L_{1}\left(K, \mu_{K}\right)$. Теперь возьмем $\varphi \in L_{1}(X, \nu)$ и рассмотрим множество

$$
\varphi_{x}(k)=\varphi\left(T_{k} x\right) \rho(k, x) .
$$

Лемма 2. Для $\nu$-почти всех $x \in X$ выполнено $\varphi_{x} \in L_{1}\left(K, \mu_{K}\right)$.

Рассмотрим произведение пространств $K \times X$, снабженное мерой $\widetilde{\nu}$, заданной формулой

$$
d \widetilde{\nu}=\rho(k, x) d \mu_{K} d \nu .
$$

Для любого фиксированного $k_{0} \in K$ имеем

$$
\int_{X} \rho\left(k_{0}, x\right) d \nu(x)=1
$$

где $\widetilde{\nu}$ - вероятностная мера.

Для любого $k \in K$ выполнено

$$
\int\left|\varphi\left(T_{k} x\right)\right| \cdot \rho(k, x) d \nu(x)=\int|\varphi(x)| d \nu(x),
$$

поэтому функция $\widetilde{\varphi}(k, x)=\varphi\left(T_{k} x\right)$ удовлетворяет условию $\widetilde{\varphi} \in L_{1}(K \times X, \widetilde{\nu})$. Теперь утверждение леммы 2 следует из теоремы Фубини.

Вернемся к доказательству леммы 1. Из условия коцикла следует, что

$$
\mathscr{A}_{K}^{\rho} \varphi(x)=\mathscr{A}_{K}^{\rho} \varphi\left(T_{k} x\right)
$$

для любого $k \in K$. Применяя теорему Фубини к пространству $K \times X$, снабженному мерой $\widetilde{\nu}$, для любого борелевского подмножества $A \subset X$ и любой $\widetilde{\varphi} \in L_{1}(K \times X, \widetilde{\nu})$ получаем

$$
\begin{aligned}
\int_{A} \int_{K} & \widetilde{\varphi}(k, x) \rho(k, x) d \mu_{K}(k) d \nu(x) \\
& =\int_{A} \int_{K} \frac{\int_{K} \widetilde{\varphi}(k, x) \rho(k, x) d \mu_{K}(k)}{\int_{K} \rho(k, x) d \mu_{K}(k)} \rho(k, x) d \mu_{K}(k) d \nu(x) .
\end{aligned}
$$

Теперь возьмем $\varphi \in L_{1}(X, \nu)$ и применим полученную формулу к функции

$$
\widetilde{\varphi}(k, x)=\varphi\left(T_{k} x\right)
$$

(отметим, что $\widetilde{\varphi} \in L_{1}(K \times X, \widetilde{\nu})$ по теореме Фубини). Получаем

$$
\int_{K}\left(\int_{A} \varphi\left(T_{k} x\right) d \nu \circ T_{k}(x)\right) d \mu_{K}(k)=\int_{K}\left(\int_{A} \mathscr{A}_{K}^{\rho} \varphi(x) d \nu \circ T_{k}(x)\right) d \mu_{K}(k) .
$$


Теперь пусть множество $A$ является $K$-инвариантным. Учитывая, что функция $\mathscr{A}_{K}^{\rho} \varphi$ также $K$-инвариантна, окончательно получаем

$$
\int_{A} \varphi(x) d \nu(x)=\int_{A} \mathscr{A}_{K}^{\rho} \varphi(x) d \nu(x),
$$

и лемма 1 полностью доказана.

2.2. Усреднение по орбитам индуктивно компактных групп. Как и ранее, пусть

$$
G=\bigcup_{n=1}^{+\infty} K(n), \quad K(n) \subset K(n+1),
$$

- индуктивно компактная группа, и пусть $\mu_{K(n)}$ - мера Хаaра на группе $K(n)$. Пусть дано измеримое действие $\mathfrak{T}$ группы $G$ на стандартном борелевском пространстве $(X, \mathscr{B})$. Пусть $\mathscr{I}_{K(n)}$ обозначает сигма-алгебру группы $K(n)$ - инвариантные измеримые подмножества в $X$, и пусть $\mathscr{I}_{G}$ - сигма-алгебра $G$ инвариантных подмножеств в $X$. Очевидно, мы имеем

$$
\mathscr{I}_{G}=\bigcap_{n=1}^{\infty} \mathscr{I}_{K(n)} \text {. }
$$

Пусть $\rho$ - положительный измеримый мультипликативный коцикл относительно действия $\mathfrak{T}$.

Определим операторы усреднения $\mathscr{A}_{K(n)}^{\rho}, n \in \mathbb{N}$, для ограниченной измеримой функции $\varphi$ на $X$ по формуле (9). Для краткости мы будем иногда писать $\mathscr{A}_{n}^{\rho}=\mathscr{A}_{K(n)}^{\rho}$.

Теперь возьмем $\nu \in \mathfrak{M}(\mathfrak{T}, \rho)$, и пусть $\mathscr{I}_{K(n)}^{\nu}, \mathscr{I}_{G}^{\nu}-$ расширения сигма-алгебр $\mathscr{I}_{K(n)}, \mathscr{I}_{G}$ относительно меры $\nu$.

Из предыдущего пункта следует, что для любой $\varphi \in L_{1}(X, \nu) \nu$-почти наверное выполнено равенство

$$
\mathscr{A}_{n}^{\rho} \varphi=\mathrm{E}\left(\varphi \mid \mathscr{I}_{K(n)}^{\nu}\right) .
$$

Поскольку $\mathscr{I}_{K(n+1)}^{\nu} \subset \mathscr{I}_{K(n)}^{\nu}$, последовательность $\mathscr{A}_{n}^{\rho} \varphi-$ обратный мартингал. Из теоремы об обратной мартингальной сходимости (см., например, [18]) следует

ПреДЛОЖеНИЕ 6. Для любой $\varphi \in L_{1}(X, \nu)$ выполнено

$$
\lim _{n \rightarrow \infty} \mathscr{A}_{n}^{\rho} \varphi=\mathrm{E}\left(\varphi \mid \mathscr{I}_{G}^{\nu}\right)
$$

как $\nu$-почти наверное, так и в $L_{1}(X, \nu)$.

Введем оператор усреднения $\mathscr{A}_{\infty}^{\rho}$, положив

$$
\mathscr{A}_{\infty}^{\rho} \varphi(x)=\lim _{n \rightarrow \infty} \mathscr{A}_{n}^{\rho} \varphi(x) .
$$

Если для данного $x \in X$ последовательность $\mathscr{A}_{n}^{\rho} \varphi(x)$ не сходится, то значение $\mathscr{A}_{\infty}^{\rho} \varphi(x)$ не определено. Из определений и теоремы об обратной мартингальной сходимости немедленно получаем 
ПреДЛОЖЕНИЕ 7. (1) Мера $\eta \in \mathfrak{M}(\mathfrak{T}, \rho)$ является эргодической тогда $и$ только тогда, когда для любой $\varphi \in L_{1}(X, \eta)$ имеем

$$
\mathscr{A}_{\infty}^{\rho} \varphi(x)=\int_{X} \varphi d \eta
$$

почти всюду относительно меры $\eta$.

(2) Обратно, если мера $\eta \in \mathfrak{M}(\mathfrak{T}, \rho)$ допускает такое плотное множество $\Psi \subset L_{1}(X, \eta)$, что для любой $\psi \in \Psi$ выполнено

$$
\mathscr{A}_{\infty}^{\rho} \psi=\int \psi d \eta
$$

почти наверное относителъно $\eta$, то мера $\eta$ является эргодической.

2.3. Эквивалентность неразложимости и эргодичности: доказательство предложения 2. Докажем сначала вспомогательное

ПредлоЖенИЕ 8. Пусть $A-G$-почти инвариантное борелевское подмножество в $X$. Тогда существует такое $G$-инвариантное множество $\widetilde{A}$, что

$$
\nu(A \triangle \widetilde{A})=0
$$

ДокАзАтеЛЬство. Пусть, как обычно, $\chi_{A}-$ индикаторная функция подмножества $A$. Если $A$ является $G$-почти инвариантным, то для почти всех $x \in A$ и всех $n \in \mathbb{N}$ имеет место равенство

$$
\mathscr{A}_{n}^{\rho} \chi_{A}(x)=1
$$

Действительно, рассмотрим множество $K(n) \times A$, наделенное произведением мер $\mu_{K(n)} \times \nu$. Для почти всех точек $(k, x) \in K(n) \times A$ имеем по определению $T_{k} x \in A$. По теореме Фубини, для почти всех $x \in X$ множество $\{k \in K(n)$ : $\left.T_{k} x \in A\right\}$ имеет полную меру, откуда $\mathscr{A}_{n}^{\rho} \chi_{A}(x)=1$.

Теперь введем множество $\widetilde{A}$ следующим образом:

$$
\widetilde{A}=\left\{x \in X: \mathscr{A}_{n}^{\rho} \chi_{A}(x)=1 \text { для всех достаточно больших } n \in \mathbb{N}\right\} \text {. }
$$

По определению, $\widetilde{A} \supset A$. С другой стороны, так как для $x \in \widetilde{A}$ выполнено $\mathscr{A}_{\infty}^{\rho} \chi_{A}(x)=1$, из равенства

$$
\int_{X} \mathscr{A}_{\infty}^{\rho} \chi_{A} d \nu=\nu(A)
$$

следует $\nu(\widetilde{A}) \leqslant \nu(A)$, откуда $\nu(\widetilde{A} \triangle A)=0$. Предложение доказано.

Предложение 2 является прямым следствием предложения 8. 
2.4. Множество эргодических мер является борелевским: доказательство предложения 4. Начнем со следующего вспомогательного предложения.

ПреДЛОЖениЕ 9. Пусть $(X, \mathscr{B})$ - стандартное борелевское пространство. Тогда существует такое счетное множество $\Phi$ ограниченных измеримых функций на $X$, что для любой вероятностной меры $\nu$ на $X$ и любой ограниченной измеримой функиии $\varphi: X \rightarrow \mathbb{R}$ существует такая последовательность $\varphi_{n} \in \Phi$, ито

(1) $\sup _{n \in \mathbb{N}, x \in X} \varphi_{n}(x)<+\infty$;

(2) $\varphi_{n} \rightarrow \varphi$ при $n \rightarrow \infty$ почти наверное относительно $\nu$.

Для доказательства достаточно взять на единичном интервале семейство кусочно-линейных функций с узлами в рациональных точках.

Перейдем к доказательству предложения 4. Ясно, что для любой фиксированной ограниченной измеримой функции $\varphi$ на $X$ множество

$$
\left\{\nu: \lim _{n \rightarrow \infty} \mathscr{A}_{n}^{\rho} \varphi \text { существует и является константой } \nu \text {-почти наверное }\right\}
$$

является борелевским. Пересекая по всем $\varphi \in \Phi$ и используя предложение 7 , получаем требуемое.

\section{§ 3. Сигма-алгебра $G$-инвариантных множеств и доказательство теоремы 1}

\section{1. Измеримые разбиения в смысле Рохлина.}

3.1.1. Пространства Лебега. Тройка $(X, \mathscr{B}, \nu)$, где $X$ - множество, $\mathscr{B}$ - сигма-алгебра на $X, \nu$ - вероятностная мера на $X$, определенная на $\mathscr{B}$ и такая, что $\mathscr{B}$ полна относительно $\nu$, называется пространством Лебега, если $X$ либо счетное, либо измеримо изоморфно единичному интервалу, снабженному сигма-алгеброй измеримых по Лебегу множеств и лебеговской мерой (возможно, со счетным семейством атомов). Мы не предполагаем наличие борелевской структуры на $X$.

3.1.2. Измеримые разбиения. Разбиение $\xi$ множества $X$ - это представление $X$ в виде дизъюнктного объединения измеримых подмножеств

$$
X=\bigsqcup X_{\alpha}
$$

Множества $X_{\alpha}$ называются элементами разбиения. Для точки $x$ элемент разбиения $\xi$, содержащий $x$, будем обозначать $\mathscr{C}_{\xi}(x)$. Семейство множеств $\Psi$ называется базисом разбиения $\xi$, если для любых двух элементов $X_{1}, X_{2}$ разбиения $\xi$ существует множество $A_{1}$ в $\Psi$, содержащее $X_{1}$ и не пересекающееся с $X_{2}$. Измеримое разбиение $\xi$ пространства $(X, \mathscr{B}, \nu)$ является по определению разбиением множества $Y \subset X$ полной меры, которое допускает счетный базис.

Следуя Рохлину [10], с измеримым разбиением $\xi$ мы ассоциируем факторпространство $\bar{X}(\xi)$, точками которого являются элементы разбиения $\xi$. Получаем естественную, почти наверное определенную проекцию $\pi_{\xi}: X \rightarrow \bar{X}(\xi)$, 
которая наделяет множество $\bar{X}(\xi)$ естественной сигма-алгеброй $\overline{\mathscr{B}}(\xi)$, спуском $\mathscr{B}$, и естественной фактормерой $\bar{\nu}_{\xi}$, спуском меры $\nu$. Рохлин доказывает, что пространство $\left(\bar{X}(\xi), \overline{\mathscr{B}}(\xi), \bar{\nu}_{\xi}\right)$ является лебеговским. Кроме того, Рохлин показывает, что мера $\nu$ допускает каноническую систему условных мер, которые определяются следующим образом. Для $\bar{\nu}_{\xi}$-почти каждого элемента $\mathscr{C}$ из разбиения $\xi$ существует такая вероятностная мера $\nu_{\mathscr{C}}$ на $\mathscr{C}$, что для любого $A \in \mathscr{B}$ функция $\operatorname{int}_{A}: \bar{X}(\xi) \rightarrow \mathbb{R}$, заданная формулой $\operatorname{int}_{A}(\mathscr{C})=\nu_{\mathscr{C}}(A)$, является $\overline{\mathscr{B}}$-измеримой и выполнено

$$
\nu(A)=\int_{\bar{X}(\xi)} \nu_{\mathscr{C}}(A) d \bar{\nu}_{\xi}(\mathscr{C}) .
$$

Эта система канонических условных мер единственна: любые две системы совпадают $\bar{\nu}_{\xi}$-почти наверное. Теперь применим к измеримому разбиению $\xi$ оператор усреднения $\mathscr{A}_{\xi}$ на $L_{1}(X, \nu)$, заданный формулой

$$
\mathscr{A}_{\xi} f(x)=\int_{\mathscr{C}_{\xi}(x)} f(x) d \nu_{\mathscr{C}_{\xi}(x)}
$$

(правая часть равенства определена $\nu$-почти наверное по теореме Рохлина [10]). Зададим измеримое разбиение $\xi$. Пусть $\mathscr{B} \xi$ - сигма-алгебра измеримых подмножеств $X$, которые являются объединением элементов $\xi$ и множества меры нуль. Рохлин доказал, что для любой $f \in L_{1}(X, \nu) \nu$-почти наверное имеет место тождество

$$
\mathrm{E}\left(f \mid \mathscr{B}_{\xi}\right)=\mathscr{A}_{\xi} f .
$$

Более того, Рохлин показал, что каждая полная сигма-подалгебра $\mathscr{B} 1 \subset \mathscr{B}$ имеет вид $\mathscr{B}_{1}=\mathscr{B}_{\xi}$ для некоторого измеримого разбиения $\xi$ пространства Лебега $(X, \mathscr{B}, \nu)$.

3.2. Борелевские разбиения. Пусть $(X, \mathscr{B})$ - стандартное борелевское пространство. Разложение

$$
X=\bigsqcup_{\alpha} X_{\alpha}
$$

где $\alpha$ принимает значения из произвольного множества индексов и где для каждого $\alpha$ множество $X_{\alpha}$ борелевское, будем называть борелевским разбиением, если существует счетное семейство

$$
Z_{1}, \ldots, Z_{n}, \ldots
$$

таких борелевских множеств, что для любых двух индексов $\alpha_{1}, \alpha_{2}$, где $\alpha_{1} \neq \alpha_{2}$, существует $i \in \mathbb{N}$, для которого выполнено

$$
X_{\alpha_{1}} \subset Z_{i}, \quad X_{\alpha_{2}} \cap Z_{i}=\varnothing .
$$

В этом случае такое счетное семейство будем называть счетным базисом разбиения.

Если $\nu$ - борелевская вероятностная мера на $X$, то пространство $(X, \mathscr{B}, \nu)$ является пространством Лебега в смысле Рохлина, а борелевское разбиение измеримым разбиением в смысле Рохлина. Заметим, что все условные меры будут в этом случае определены на борелевской сигма-алгебре. 
3.3. Измеримое разбиение, соответствующее сигма-алгебре инвариантных множеств. Нашей первой целью будет явное описание измеримого разбиения, соответствующего сигма-алгебре $\mathscr{I}_{G} G$-инвариантных множеств.

Пусть $\Phi-$ множество из предложения 9 и $\Phi=\left\{\varphi_{1}, \varphi_{2}, \ldots, \varphi_{n}, \ldots\right\}$. Зададим множество $X(\Phi, \rho)$ формулой

$$
X(\Phi, \rho)=\left\{x \in X: A_{\infty}^{\rho} \varphi_{k}(x) \text { определены для всех } k \in \mathbb{N}\right\} .
$$

Множество $X(\Phi, \rho)$, очевидно, является борелевским. Заметим, что для любой $\nu \in \mathfrak{M}(\mathfrak{T}, \rho)$ имеем

$$
\nu(\mathfrak{M}(\mathfrak{T}, \rho))=1 .
$$

Пусть $\mathbb{R}^{\mathbb{N}}$ - пространство всех вещественных последовательностей

$$
\mathbb{R}^{\mathbb{N}}=\left\{\mathbf{r}=\left(r_{k}\right), k \in \mathbb{N}, r_{k} \in \mathbb{R}\right\} .
$$

Мы снабдим $\mathbb{R}^{\mathbb{N}}$ обычной сигма-алгеброй, которая превратит его в стандартное борелевское пространство. Для $\mathbf{r} \in \mathbb{R}^{\mathbb{N}}$ зададим подмножество $X(\mathbf{r}, \Phi, \rho)$ формулой

$$
X(\mathbf{r}, \Phi, \rho)=\left\{x \in X(\Phi, \rho): A_{\infty}^{\rho} \varphi_{k}(x)=r_{k}, k \in \mathbb{N}\right\} .
$$

Для любого $\mathbf{r} \in \mathbb{R}^{\mathbb{N}}$ множество $X(\mathbf{r}, \Phi, \rho)$ является борелевским, и мы, очевидно, имеем

$$
X(\Phi, \rho)=\bigsqcup_{\mathbf{r} \in \mathbb{R}^{\mathbb{N}}} X(\mathbf{r}, \Phi, \rho) .
$$

Из определения следует, что борелевское разбиение

$$
X=(X \backslash X(\Phi, \rho)) \sqcup \bigsqcup_{\mathbf{r} \in \mathbb{R}^{\mathbb{N}}} X(\mathbf{r}, \Phi, \rho)
$$

имеет счетный базис.

Введем отображение

$$
\Pi_{\Phi}: X(\Phi, \rho) \rightarrow \mathbb{R}^{\mathbb{N}}
$$

формулой

$$
\Pi_{\Phi}(x)=\left(\mathscr{A}_{\infty}^{\rho} \varphi_{1}(x), \ldots, \mathscr{A}_{\infty}^{\rho} \varphi_{n}(x), \ldots\right) .
$$

Отображение $\Pi_{\Phi}$ является борелевским по определению. Далее, определим отображение

$$
\operatorname{Int}_{\Phi}: \mathfrak{M}(\mathfrak{T}, \rho) \rightarrow \mathbb{R}^{\mathbb{N}}
$$

формулой

$$
\operatorname{Int}_{\Phi}(\nu)=\left(\int_{X} \varphi_{1} d \nu, \ldots, \int_{X} \varphi_{n} d \nu, \ldots\right)
$$

Отображение Int $\Phi$ также по определению борелевское и инъективное.

По теореме Суслина (см. [19]-[21]) множества $\operatorname{Int}_{\Phi}(\mathfrak{M}(\mathfrak{T}, \rho))$ и $\operatorname{Int}_{\Phi}(\mathfrak{M} \operatorname{erg}(\mathfrak{T}, \rho))$ являются борелевскими. Зададим подмножество $X_{\mathrm{erg}} \subset X$ формулой

$$
X_{\mathrm{erg}}=\Pi_{\Phi}^{-1}\left(\operatorname{Int}_{\Phi}\left(\mathfrak{M}_{\operatorname{erg}}(\mathfrak{T}, \rho)\right)\right)
$$


Снова из теоремы Суслина следует, что множество $X_{\mathrm{erg}}$ борелевское. Таким образом, мы получаем следующую диаграмму, все стрелки которой соответствуют борелевским отображениям:

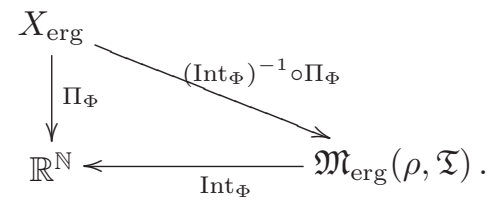

Теперь мы видим, что для любой $\nu \in \mathfrak{M}(\mathfrak{T}, \rho)$ выполнено

$$
\nu\left(X_{\mathrm{erg}}\right)=1
$$

Действительно, рассмотрим произвольную $\nu \in \mathfrak{M}(\mathfrak{T}, \rho)$. Борелевское разбиение $\xi$ индуцирует измеримое разбиение, которое мы обозначим $\xi^{\nu}$. Пусть $\bar{X}\left(\xi^{\nu}\right)$ - пространство элементов разбиения $\xi$, или, другими словами, факторпространство множества $X$ по разбиению $\xi$. Пусть

$$
\pi_{\xi^{\nu}}: X \rightarrow \bar{X}\left(\xi^{\nu}\right)
$$

- естественная проекция и

$$
\widetilde{\nu}=\left(\pi_{\xi^{\nu}}\right)_{*} \nu
$$

- фактормера на $\bar{X}\left(\xi^{\nu}\right)$.

По теореме Рохлина $\widetilde{\nu}$-почти каждый элемент $\mathscr{C}$ разбиения $\xi^{\nu}$ несет каноническую условную меру $\nu_{\mathscr{C}}$. Следующее предложение является ключевым шагом в построении эргодического разложения.

ПРЕДЛОЖЕНИЕ 10. 1) Измеримое разбиение $\xi^{\nu}$ порождает сигма-алгебpy $\mathscr{I}_{G}^{\nu}$, m.е. $\nu$-пополнение сигма-алгебрь борелевских G-инвариантных множеств.

2) Для $\widetilde{\nu}$-почти каждого $\mathscr{C}$ имеем $\nu_{\mathscr{C}} \in \mathfrak{M}_{\operatorname{erg}}(\rho, \mathfrak{T})$.

Предложение будет доказано в следующем пункте. Для получения эргодического разложения меры $\nu$ будем использовать разложение Рохлина

$$
\nu=\int_{\bar{X}\left(\xi^{\nu}\right)} \nu \mathscr{C} d \widetilde{\nu}(\mathscr{C}) .
$$

Пусть отображение

$$
\operatorname{mes}_{\xi^{\nu}}: \bar{X}\left(\xi^{\nu}\right) \rightarrow \mathfrak{M}_{\operatorname{erg}}(\rho, \mathfrak{T})
$$

задано формулой

$$
\operatorname{mes}_{\xi^{\nu}}(\mathscr{C})=\nu \mathscr{C} .
$$


ПрЕДЛОЖЕНИЕ 11. Отображение $\operatorname{mes}_{\xi^{\nu}} \widetilde{\nu}$-измеримо.

ДокАзАТЕЛьство. Пусть $\varphi$ - ограниченная измеримая функция на $X$ и $\alpha \in \mathbb{R}$. По определению измеримой структуры на факторпространстве $\bar{X}\left(\xi^{\nu}\right)$ достаточно показать, что множество

$$
\left\{x \in X: \int \varphi d \nu_{\mathscr{C}(x)}>\alpha\right\}
$$

является $\nu$-измеримым. Но из предложения 10 мы имеем $\nu$-почти наверное равенство

$$
\left\{x \in X: \int \varphi d \nu_{\mathscr{C}(x)}>\alpha\right\}=\left\{x \in X: \mathscr{A}_{\infty}^{\rho} \varphi(x)>\alpha\right\} .
$$

Поскольку множество $\left\{x \in X: \mathscr{A}_{\infty}^{\rho} \varphi(x)>\alpha\right\}$ борелевское, предложение доказано.

Для $x \in X$ обозначим через $\mathscr{C}_{\xi}(x)$ элемент разбиения $\xi$, содержащий $x$, и зададим отображение

$$
\operatorname{Mes}_{\xi^{\nu}}: X \rightarrow \mathfrak{M}_{\operatorname{erg}}(\rho, \mathfrak{T})
$$

формулой

$$
\operatorname{Mes}_{\xi^{\nu}}(x)=\nu_{\mathscr{C}_{\xi}(x)} .
$$

Получаем коммутативную диаграмму

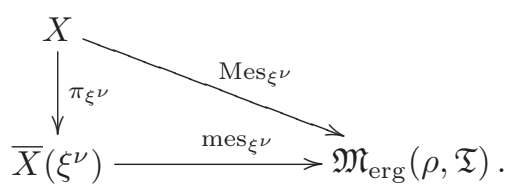

В частности, отображение $\operatorname{Mes}_{\xi^{\nu}}$ ע-измеримо. Из предложения 10 немедленно вытекает

СлеДСтвиЕ 4. Для любого $\nu \in M_{\mathrm{erg}}(\mathfrak{T}, \rho)$ выполнено $\nu\left(X_{\mathrm{erg}}\right)=1$. Равенcm80

$$
\operatorname{Mes}_{\xi^{\nu}}=\left(\operatorname{Int}_{\Phi}\right)^{-1} \circ \Pi_{\Phi}
$$

сохраняется $\nu$-почти наверное.

Замечая, что

$$
\bar{\nu}=\left(\operatorname{Mes}_{\xi^{\nu}}\right)_{*} \nu=\left(\operatorname{mes}_{\xi^{\nu}}\right)_{*} \widetilde{\nu},
$$

мы получим эргодическое разложение

$$
\nu=\int_{\mathfrak{M}_{\operatorname{erg}}(\rho, \mathfrak{T})} \eta d \bar{\nu}(\eta)
$$

меры $\nu$. Для завершения доказательства первых двух утверждений теоремы 1 остается доказать предложение 10. 


\section{4. Доказательство предложения 10.}

3.4.1. Доказательство утверждения 1). С одной стороны, каждый элемент разбиения $\xi^{\nu}$ является по определению $G$-инвариантным множеством.

Обратно, пусть $A$ - это $G$-инвариантное множество. Нам надо найти измеримое множество $A^{\prime}$, которое является семейством элементов разбиения $\xi^{\nu}$ и удовлетворяет равенству

$$
\nu\left(A \triangle A^{\prime}\right)=0
$$

Возьмем такую последовательность $\varphi_{n_{k}} \in \Phi$, что

$$
\sup _{k \in \mathbb{N}, x \in X} \varphi_{n_{k}}(x)<+\infty
$$

и $\varphi_{n_{k}} \rightarrow \chi_{A} \nu$-почти наверное при $k \rightarrow \infty$.

Пусть теперь

$$
\begin{gathered}
R_{A}=\left\{\mathbf{r} \in \mathbb{R}^{\mathbb{N}}, \mathbf{r}=\left(r_{n}\right), \lim _{k \rightarrow \infty} r_{n_{k}}=1\right\} \\
A^{\prime}=\bigcup_{\mathbf{r} \in R_{A}} X(\rho, \Phi, \mathbf{r}), \quad A^{\prime \prime}=\left\{x \in X: \mathscr{A}_{\infty}^{\rho} \chi_{A}(x)=1\right\} .
\end{gathered}
$$

Поскольку $A$ - это $G$-инвариантное множество, имеем

$$
\nu\left(A \triangle A^{\prime \prime}\right)=0
$$

Так как

$$
\lim _{k \rightarrow \infty} \varphi_{n_{k}}=\chi_{A}
$$

$\nu$-почти наверное и все функции равномерно ограничены, имеем

$$
\mathscr{A}_{\infty}^{\rho} \varphi_{n_{k}} \rightarrow \mathscr{A}_{\infty}^{\rho} \chi_{A}
$$

почти наверное при $k \rightarrow \infty$. Из этого следует, что

$$
\nu\left(A^{\prime} \triangle A^{\prime \prime}\right)=0
$$

и, наконец, получаем

$$
\nu\left(A \triangle A^{\prime}\right)=0
$$

что и требовалось доказать.

3.4.2. Доказательство утверждения 2) предложения 10.

ПреДЛОЖениЕ 12. Для каждого $g \in G$, для $\bar{\nu}$-почти каждого $\mathscr{C} \in \bar{X}\left(\xi^{\nu}\right) u$ $\nu_{\mathscr{C}}$-почти каждого $x \in X$ имеем

$$
\frac{d \nu_{\mathscr{C}} \circ T_{g}}{d \nu_{\mathscr{C}}}(x)=\rho(g, x) .
$$


ДоказАТЕЛЬСтво. Это непосредственно следует из единственности канонической системы условных мер. В самом деле, с одной стороны,

$$
\nu \circ T_{g}=\int_{\bar{X}\left(\xi^{\nu}\right)} \nu_{\mathscr{C}} \circ T_{g} d \bar{\nu}(\mathscr{C})
$$

с другой стороны,

$$
\nu \circ T_{g}=\rho(g, x) \cdot \nu=\int_{\bar{X}\left(\xi^{\nu}\right)} \rho(g, x) \cdot \nu_{\mathscr{C}} d \bar{\nu}(\mathscr{C}),
$$

откуда $\nu_{\mathscr{C}} \cdot T_{g}=\rho(g, x) \cdot \nu_{\mathscr{C}}$ для $\widetilde{\nu}$-почти всех $\mathscr{C} \in \bar{X}\left(\xi^{\nu}\right)$, и предложение доказано.

Послойная непрерывность коцикла необходима для того, чтобы перейти от счетной плотной подгруппы ко всей группе.

ПреДЛОЖЕНИЕ 13. Пусть $\rho$ - положителъный борелевский послойно непрерывный коиикл над измеримым действием $\mathfrak{T}_{K}$ компактной группь $K$ на стандартном борелевском пространстве $(X, \mathscr{B})$. Пусть $\nu$ - борелевская вероятностная мера на $X$. Рассмотрим плотное подмножество $K^{\prime} \subset K u$ предположим, что равенство

$$
\frac{d \nu \circ T_{k}}{d \nu}=\rho(k, x)
$$

верно для всех $k \in K^{\prime}$. Тогда $\nu \in \mathfrak{M}\left(\mathfrak{T}_{K}, \rho\right)$.

ДокАЗАТЕЛЬСтво. Применим теорему Варадараяна о реализации к действию нашей компактной группы $K$. Перейдя, если необходимо, к большему пространству, построенному в теореме, мы можем считать, что $X$ - компактное метрическое пространство, $\nu$ - борелевская вероятностная мера и действие группы $K$ на $X$ непрерывно. Следовательно, если $k_{n} \rightarrow k_{\infty}$ в $K$ при $n \rightarrow \infty$, то

$$
\nu \circ T_{k_{n}} \rightarrow \nu \circ T_{k_{\infty}}
$$

слабо в пространстве борелевских вероятностных мер на $X$. Остается показать, что меры $\nu=\rho\left(k_{n}, x\right) \cdot \nu$ слабо сходятся к мере $\rho(k, x) \cdot \nu$ при $n \rightarrow \infty$, и равенство $\nu \circ T_{k_{\infty}}=\rho\left(k_{\infty}, x\right) \cdot \nu$ будет доказано. Прежде всего, докажем, что функция

$$
\rho_{\max }(x)=\max _{k \in K} \rho(k, x)
$$

определена и измерима в $X$ (поскольку, по непрерывности, максимум можно заменить супремумом на счетном плотном подмножестве). Мы покажем, что для любой ограниченной измеримой функции $\psi$ на $X$ выполнено

$$
\lim _{n \rightarrow \infty} \int_{X} \psi(x) \rho\left(k_{n}, x\right) d \nu(x)=\int_{X} \psi(x) \rho\left(k_{\infty}, x\right) d \nu(x) .
$$

Допустим, что $\psi$ удовлетворяет неравенству $0 \leqslant \psi \leqslant 1$. Для каждого $x \in X$ выполнено

$$
\lim _{n \rightarrow \infty} \rho\left(k_{n}, x\right)=\rho\left(k_{\infty}, x\right) .
$$


По лемме Фату

$$
\int \psi(x) \rho\left(k_{\infty}, x\right) d \nu(x) \leqslant \liminf _{n \rightarrow \infty} \int \psi(x) \rho\left(k_{n}, x\right) d \nu(x) .
$$

Для $N>0$ определим $X_{N}=\left\{x: \rho_{\max }(x) \leqslant N\right\}$. Возьмем $\varepsilon>0$ и выберем $N$ настолько большое, чтобы выполнялось

$$
\nu\left(X \backslash X_{N}\right)<\varepsilon, \quad \int_{X \backslash X_{N}} \psi(x) \rho\left(k_{\infty}, x\right) d \nu(x)<\varepsilon .
$$

Заметим, что поскольку $X_{N} K$-инвариантен, для всех $n \in \mathbb{N}$ выполнено

$$
\int_{X \backslash X_{N}} \psi(x) \rho\left(k_{n}, x\right) d \nu(x) \leqslant \nu \circ T_{k_{n}}\left(X \backslash X_{N}\right)=\nu\left(X \backslash X_{N}\right)<\varepsilon .
$$

По теореме об ограниченной сходимости мы имеем

$$
\lim _{n \rightarrow \infty} \int_{X_{N}} \psi(x) \rho\left(k_{n}, x\right) d \nu(x)=\int_{X_{N}} \psi(x) \rho\left(k_{\infty}, x\right) d \nu(x),
$$

откуда

$$
\int_{X} \psi(x) \rho\left(k_{\infty}, x\right) d \nu(x) \geqslant \limsup _{n \rightarrow \infty} \int \psi(x) \rho\left(k_{n}, x\right) d \nu(x)-3 \varepsilon .
$$

Так как $\varepsilon$ произвольно, предложение 13 доказано.

Вернемся к доказательству утверждения 2) предложения 10.

Возьмем $n_{0} \in \mathbb{N}$ и покажем, что для $\widetilde{\nu}$-почти каждого $\mathscr{C}$ и всех $k \in K\left(n_{0}\right)$ выполнено

$$
\frac{d \nu_{\mathscr{C}} \circ T_{k}}{d \nu_{\mathscr{C}}}=\rho(k, x)
$$

Выберем счетную плотную подгруппу $K^{\prime} \subset K\left(n_{0}\right)$. Равенство (17) верно для всех $k \in K^{\prime}$ и $\widetilde{\nu}$-почти всех $\mathscr{C}$. Но тогда из послойной непрерывности коцикла $\rho$ следует, что (17) верно также для всех $k \in K\left(n_{0}\right)$. Следовательно, $\nu_{\mathscr{C}} \in \mathfrak{M}(\rho, \mathfrak{T})$ для $\widetilde{\nu}$-почти всех $\mathscr{C}$. Далее, по определению разбиения $\xi$ для каждого $\varphi \in \Phi$ выполнено

$$
\mathscr{A}_{\infty}^{\rho}=\int \varphi d \nu_{\mathscr{C}}
$$

$\nu_{\mathscr{C}}$-почти наверное (действительно, функция $\mathscr{A}_{\infty}^{\rho} \varphi$ является почти всегда константой в ограничении на $\mathscr{C}$, но, с другой стороны, константа должна быть равна средней величине).

Так как $\Phi$ плотно в $L_{1}\left(X, \nu_{\mathscr{C}}\right)$ и $\nu_{\mathscr{C}} \in \mathfrak{M}(\mathfrak{T}, \rho)$, мы делаем вывод, что $\nu_{\mathscr{C}}$ является эргодическим для $\widetilde{\nu}$-почти каждого $\mathscr{C}$, и предложение 10 полностью доказано. 
3.5. Единственность эргодического разложения. Рассмотрим отображение

$$
\text { Mes: } \mathfrak{M}(\mathfrak{T}, \rho) \rightarrow \mathfrak{M}\left(\mathfrak{M}_{\mathrm{erg}}(\mathfrak{T}, \rho)\right),
$$

которое мере $\nu \in \mathfrak{M}(\mathfrak{T}, \rho)$ ставит в соответствие меру

$$
\bar{\nu}=\operatorname{Mes}(\nu)=\left(\operatorname{Mes}_{\xi^{\nu}}\right)_{*} \nu
$$

По определению имеем

$$
\nu=\int_{\mathfrak{M}_{\operatorname{erg}}(\mathfrak{T}, \rho)} \eta d \bar{\nu}(\eta) .
$$

Обратно, зададим отображение $\mathrm{ED}: \mathfrak{M}\left(\mathfrak{M}_{\operatorname{erg}}(\mathfrak{T}, \rho)\right) \rightarrow \mathfrak{M}(\mathfrak{T}, \rho)$, которое переводит меру $\bar{\nu} \in \mathfrak{M}\left(\mathfrak{M}_{\operatorname{erg}}(\mathfrak{T}, \rho)\right)$ в меру $\nu$ по формуле $(18)$.

Мы должны проверить, что отображения ED и Mes измеримы по Борелю и являются взаимно обратными. Из определения ясно, что отображение ED является измеримым по Борелю и $\mathrm{ED} \circ \mathrm{Mes}=\mathrm{Id}$. Перейдем к доказательству остальных утверждений.

Сначала проверим, что отображение Mes измеримо по Борелю. Действительно, возьмем $\alpha_{1}, \alpha_{2} \in \mathbb{R}$, множество $A \in \mathscr{B}(X)$ и рассмотрим множество $\widetilde{A}_{\alpha_{1}, \alpha_{2}} \subset \mathfrak{M}\left(\mathfrak{M}_{\mathrm{erg}}(\mathfrak{T}, \rho)\right)$, заданное формулой

$$
\widetilde{A}_{\alpha_{1}, \alpha_{2}}=\left\{\bar{\nu} \in \mathfrak{M}\left(\mathfrak{M}_{\operatorname{erg}}(\mathfrak{T}, \rho)\right): \bar{\nu}\left(\left\{\eta \in \mathfrak{M}_{\operatorname{erg}}(\mathfrak{T}, \rho): \eta(A)>\alpha_{1}\right\}\right)>\alpha_{2}\right\} .
$$

Ясно, что

$$
(\mathrm{Mes})^{-1}\left(\widetilde{A}_{\alpha_{1}, \alpha_{2}}\right)=\left\{\nu \in \mathfrak{M}(\mathfrak{T}, \rho): \nu\left(\left\{x \in X: \mathscr{A}_{\infty}^{\rho} \chi_{A}(x)>\alpha_{1}\right\}\right)>\alpha_{2}\right\},
$$

и измеримость отображения Mes доказана.

Остается проверить, что для данной меры $\nu \in \mathfrak{M}(\mathfrak{T}, \rho)$ существует только одна такая мера $\bar{\nu} \in \mathfrak{M}\left(\mathfrak{M}_{\mathrm{erg}}(\mathfrak{T}, \rho)\right)$, что $\nu=\operatorname{ED}(\bar{\nu})$, а именно $\bar{\nu}=\operatorname{Mes}(\nu)$. Для доказательства обратимости отображения ED достаточно установить следующее

ПРЕДЛОЖЕНИЕ 14. Пустъ $\bar{\nu}_{1}, \bar{\nu}_{2} \in \mathfrak{M}\left(\mathfrak{M}_{\operatorname{erg}}(\mathfrak{T}, \rho)\right)$. Если $\bar{\nu}_{1} \perp \bar{\nu}_{2}$, mo

$$
\operatorname{ED}\left(\bar{\nu}_{1}\right) \perp \operatorname{ED}\left(\bar{\nu}_{2}\right) .
$$

ДокАЗАтельство. Пусть $\nu_{0}=\mathrm{ED}\left(\left(\bar{\nu}_{1}+\bar{\nu}_{2}\right) / 2\right)$ и $A_{1}, A_{2} \subset \mathfrak{M}\left(\mathfrak{M}_{\operatorname{erg}}(\mathfrak{T}, \rho)\right)-$ непересекающиеся множества, удовлетворяющие равенствам

$$
\bar{\nu}_{1}\left(A_{1}\right)=\bar{\nu}_{2}\left(A_{2}\right)=1, \quad \bar{\nu}_{1}\left(A_{2}\right)=\bar{\nu}_{2}\left(A_{1}\right)=0 .
$$

Тогда множества $X_{1}=\left(\operatorname{Mes}_{\xi_{0}}\right)^{-1}\left(A_{1}\right), X_{2}=\left(\operatorname{Mes}_{\xi^{\nu_{0}}}\right)^{-1}\left(A_{2}\right)$ не пересекаются и $\nu_{0}$-измеримы. Более того, по определению

$$
\operatorname{ED}\left(\bar{\nu}_{1}\right)\left(X_{1}\right)=\operatorname{ED}\left(\bar{\nu}_{2}\right)\left(X_{2}\right)=1, \quad \operatorname{ED}\left(\bar{\nu}_{1}\right)\left(X_{2}\right)=\operatorname{ED}\left(\bar{\nu}_{2}\right)\left(X_{1}\right)=0,
$$

и тем самым предложение доказано. 
Единственность эргодического разложения - утверждение (3) теоремы 1 полностью доказано. Утверждение (4) теоремы 1 также обосновано: действительно, если $\nu_{1}$ абсолютно непрерывна по мере $\nu_{2}$, то по определению множества меры нуль относительно $\nu_{2}$ также должны иметь меру нуль относительно $\nu_{1}$, тем более это верно для инвариантных множеств, и, следовательно, по построению, для разложимых мер также верно, что $\bar{\nu}_{1}$ абсолютно непрерывна относительно $\bar{\nu}_{2}$. Обратно, если $\bar{\nu}_{1}$ абсолютно непрерывна относительно $\bar{\nu}_{2}$, то по определению эргодического разложения $\nu_{1}$ абсолютно непрерывна относительно $\nu_{2}$. Снова применяя предложение 14 , мы завершаем доказательство утверждения (4) теоремы 1.

Теорема 1 полностью доказана.

\section{§ 4. Редукция бесконечных мер к конечным мерам}

4.1. Доказательство теоремы 2. В доказательстве следствия 2 мы построили эргодическое разложение

$$
\nu=\int_{\mathfrak{M}_{f, 1, \operatorname{erg}}^{\infty}} \eta d \widetilde{\nu}(\eta)
$$

где мера $\widetilde{\nu} \in \mathfrak{M}\left(\mathfrak{M}_{f, 1, \mathrm{erg}}^{\infty}\right)$ автоматически является допустимой.

Взяв произвольную положительную измеримую функцию $\varphi: \mathbb{P M}^{\infty} \rightarrow \mathbb{R}_{>0}$, мы можем видоизменить разложение, записав (19) в виде

$$
\nu=\int_{\mathfrak{M}_{f, 1, \mathrm{erg}}^{\infty}} \frac{\eta}{\varphi(\mathbf{p}(\eta))} \varphi(\mathbf{p}(\eta)) d \widetilde{\nu}(\eta) .
$$

Обратно, для любой $\sigma$-конечной меры $\nu^{\prime} \in \mathfrak{M}^{\infty}\left(\mathbb{P} \mathfrak{M}^{\infty}\right)$, удовлетворяющей условию $\left[\nu^{\prime}\right]=\left[\mathbf{p}_{*} \widetilde{\nu}\right]$, мы можем немедленно предъявить меру $\check{\nu} \in \mathfrak{M}^{\infty}\left(\mathfrak{M}^{\infty}\right)$ такую, что $\mathbf{p}_{*} \check{\nu}=\widetilde{\nu}$ и

$$
\nu=\int_{\mathfrak{M} \infty} \eta d \check{\nu}(\eta) .
$$

Так как $\widetilde{\nu}$ допустима, мера $\check{\nu}$ с заданными свойствами единственна.

Для завершения доказательства мы должны показать, что класс $\left[\mathbf{p}_{*} \bar{\nu}\right]$ один и тот же для всех допустимых мер $\bar{\nu}$, встречающихся в эргодическом разложении данной меры $\nu$.

Напомним, что отображение $P_{f}: \mathfrak{M}_{f}^{\infty} \rightarrow \mathfrak{M}$ было задано формулой

$$
P_{f}(\nu)=\frac{f \nu}{\nu(f)} .
$$

Для $\lambda \in \mathbb{R}_{+}$мы, очевидно, имеем

$$
P_{f}(\lambda \nu)=P_{f}(\nu)
$$

Поэтому отображение $P_{f}$ порождает отображение из $\mathbb{P}_{f}^{\infty}$ в $\mathfrak{M}$, для которого мы будем использовать те же обозначения. 
Отображение $P_{f}: \mathbb{P}_{f}^{\infty} \rightarrow \mathfrak{M}$ обратимо: обратным является отображение, которое мере $\nu \in \mathfrak{M}$ ставит в соответствие проективный класс эквивалентности меры $\nu / f$.

Рассмотрим произвольное эргодическое разложение

$$
\nu=\int_{\mathfrak{M} \infty} \eta d \widetilde{\nu}(\eta)
$$

меры $\nu \in \mathfrak{M}_{f}^{\infty}$. По определению для меры $\widetilde{\nu} \in \mathfrak{M}\left(\mathfrak{M}^{\infty}\right)$ выполнено

$$
\widetilde{\nu}\left(\mathfrak{M}_{f, \text { erg }}^{\infty}\right)=1 .
$$

Поэтому возьмем эргодическое разложение

$$
\nu=\int_{\mathfrak{M}_{\mathrm{erg}, g}^{\infty}} \eta d \widetilde{\nu}(\eta) .
$$

Применяя отображение $P_{f}$, получаем

$$
P_{f} \nu=\int_{\mathfrak{M}_{\mathrm{erg}, f}^{\infty}} P_{f} \eta \cdot \frac{\eta(f)}{\nu(f)} d \widetilde{\nu}(\eta) .
$$

Mepa

$$
\frac{\eta(f)}{\nu(f)} d \widetilde{\nu}(\eta)
$$

является вероятностной мерой на $\mathfrak{M}_{\mathrm{erg}, f}^{\infty}$, поскольку такова $P_{f} \eta$ для любой $\eta \in \mathfrak{M}_{f}^{\infty}$.

Зададим меру $\check{\nu} \in \mathfrak{M}\left(\mathfrak{M}_{\mathrm{erg}, f}^{\infty}\right)$ формулой

$$
d \check{\nu}(\eta)=\frac{\eta(f)}{\nu(f)} d \widetilde{\nu}(\eta)
$$

и перепишем (22) в виде

$$
P_{f} \nu=\int_{\mathfrak{M}} \eta d\left(\left(P_{f}\right)_{*} \check{\nu}\right) .
$$

По определению формула (23) задает эргодическое разложение меры $P_{f} \nu \in$ $\mathfrak{M}\left(\mathfrak{T}, \rho_{f}\right)$. В самом деле, мера $\left(P_{f}\right)_{*} \check{\nu}$ по определению сосредоточена на $\mathfrak{M}_{\mathrm{erg}}\left(\mathfrak{T}, \rho_{f}\right)$. Так как эргодическое разложение единственно в $\mathfrak{M}\left(\mathfrak{T}, \rho_{f}\right)$, мы получаем, что мера $\left(P_{f}\right)_{*} \check{\nu}$ не зависит от выбора исходного эргодического разложения (21).

Из очевидного равенства $[\check{\nu}]=[\widetilde{\nu}]$ следует, что

$$
\left[\left(P_{f}\right)_{*} \check{\nu}\right]=\left[\left(P_{f}\right)_{*} \widetilde{\nu}\right]
$$

и тем самым класс $\left[\left(P_{f}\right)_{*} \widetilde{\nu}\right]$ не зависит от выбора эргодического разложения (21).

Вспомним теперь, что отображение $P_{f}$ индуцирует борелевский изоморфизм между борелевскими пространствами $\mathbb{P}_{\mathfrak{f}}^{\infty}$ и $\mathfrak{M}$. Так как класс $\left[\left(P_{f}\right)_{*} \widetilde{\nu}\right]$ не зависит от выбора эргодического разложения, это же верно и для класса $\left[\mathbf{p}_{*} \widetilde{\nu}\right]$.

Теорема 2 полностью доказана. 
4.2. Конечные и бесконечные эргодические компоненты. Эргодические компоненты бесконечной $G$-инвариантной меры могут быть как конечными, так и бесконечными. Из предыдущих результатов вытекает следующее описание множеств, на которых сосредоточены конечные и бесконечные эргодические компоненты.

СЛЕДСТвИЕ 5. Пусть Т - измеримое действие индуктивно компактной группы $G$ на стандартном борелевском пространстве $(X, \mathscr{B})$ и $\nu$ - такая $\sigma$ конечная T-инвариантная борелевская мера на $X$, что пространство $L_{1}(X, \nu)$ содержит положительную измеримую по Борелю послойно непрерывную функцию. Тогда существуют непересекающиеся борелевские $G$-инвариантные подмножества $X_{1}, X_{2}$ множества $X$, для которых $X_{1} \cup X_{2}=X$, и выполнены следующие условия.

(1) Существует такое семейство $Y_{n}$ борелевских $G$-инвариантных подмножеств, что $\nu\left(Y_{n}\right)<+\infty$ u

$$
X_{1}=\bigcup_{n} Y_{n}
$$

Если $Y$ - борелевское $G$-инвариантное подмножество, удовлетворяющее условию $\nu(Y)<+\infty$, то $\nu\left(X_{1} \backslash Y\right)=0$. Относительно любого эргодического разложения почти все эргодические компоненты меры $\left.\nu\right|_{X_{1}}$ являются конечными.

(2) Если $\varphi$ - ограниченная измеримая функиия, сосредоточенная на $\mathrm{X}_{2}$ u интегрируемая с квадратом по мере $\nu$, то для соответствующей последовательности усреднений выполнено $\mathscr{A}_{n} \varphi \rightarrow 0$ в $L_{2}(X, \nu)$. Относительно любого эргодического разложения почти все эргодические компоненты меры $\left.\nu\right|_{X_{2}}$ являются бесконечными.

По определению множества $X_{1}, X_{2}$ определены однозначно с точностью до подмножеств меры нуль.

В случае непрерывных действий можно дать следующее описание. Пусть $X$ - полное сепарабельное метрическое пространство и $\nu$ - борелевская мера, сопоставляющая конечный вес каждому шару. Возьмем точку $x \in X$ и зададим орбиталъные меры $\eta_{x}^{n}$ формулой

$$
\eta_{x}^{n}=\int_{K(n)} \delta_{T_{k} x} d \mu_{K(n)}(k) .
$$

Эквивалентно, для любой ограниченной непрерывной функции $f$ на $X$ выполнено

$$
\int_{X} f d \eta_{x}^{n}=\int_{K(n)} f\left(T_{k} x\right) d \mu_{K(n)}(k) .
$$

В этом случае множества $X_{1}, X_{2}$ могут быть охарактеризованы следующим образом: множество $X_{1}$ - это множество всех $x \in X$, для которых последовательность $\eta_{x}^{n}$ слабо сходится к вероятностной мере при $n \rightarrow \infty$, в то время как 
$X_{2}$ - это множество всех таких $x \in X$, что для любой ограниченной непрерывной функции $f$ на $X$, носитель которой - ограниченное множество, выполнено

$$
\lim _{n \rightarrow \infty} \int_{X} f d \eta_{x}^{n}=0
$$

\section{§5. Пример Колмогорова и доказательство предложения 1}

5.1. Пример Колмогорова. Для полноты изложения мы кратко напомним пример Колмогорова [1]. Он показывает, что для действий больших групп эргодические инвариантные вероятностные меры могут не быть неразложимыми.

Пусть $G$ - группа всех биекций на $\mathbb{Z}$ и $\Omega_{2}$ - пространство бесконечных в обе стороны бинарных последовательностей. Группа $G$ действует на $\Omega_{2}$ и сохраняет там любую меру Бернулли.

Пусть $G_{0} \subset G$ - подгруппа конечных перестановок, т.е. перестановок, в которых перемещается только конечное подмножество символов. Группа $G_{0}$ является индуктивно компактной. Теорема де Финетти утверждает, что $G_{0}$-инвариантные неразложимые (или, эквивалентно, эргодические) вероятностные меры на $\Omega_{2}$ являются мерами Бернулли.

Из этого следует, что $G$-инвариантные неразложимые вероятностные меры - это также в точности меры Бернулли. Однако если $\nu_{1}$ и $\nu_{2}$ - две различные неатомические меры Бернулли на $\Omega_{2}$, то мера $\left(\nu_{1}+\nu_{2}\right) / 2$ является эргодической! Действительно, группа $G$ имеет только счетное число орбит на $\Omega_{2}$ и легко проверить, что любое $G$-инвариантное множество должно иметь либо полную, либо нулевую меру относительно $\left(\nu_{1}+\nu_{2}\right) / 2$.

5.2. Доказательство предложения 1. Пусть, как и ранее, $(X, \mathscr{B})$ - стандартное борелевское пространство. Пусть $G$ - произвольная группа и $\mathfrak{T}$ - действие группы $G$ на $X$. Будем называть действие $\mathfrak{T}$ слабо измеримым, если для любого $g \in G$ преобразование $T_{g}$ измеримо по Борелю. Аналогично, положительный мультипликативный коцикл

$$
\rho: G \times X \rightarrow \mathbb{R}_{>0}
$$

будем называть слабо измеримым, если для любого $g \in G$ функция $\rho(g, x)$ является измеримой по Борелю в точке $x$. Для слабо измеримого коцикла $\rho$ пространство $\mathfrak{M}(\mathfrak{T}, \rho)$ определяется аналогичным образом и снова является выпуклым конусом. Будем называть меру $\nu \in \mathfrak{M}(\mathfrak{T}, \rho)$ сильно неразложимой, если представление

$$
\nu=\alpha \nu_{1}+(1-\alpha) \nu_{2},
$$

где $\nu_{1}, \nu_{2} \in \mathfrak{M}(\mathfrak{T}, \rho), \alpha \in(0,1)$, возможно, только когда $\nu=\nu_{1}=\nu_{2}$. Будем называть меру $\nu$ слабо неразложимой, если для любого измеримого по Борелю множества $A$, удовлетворяющего для каждого $g \in G$ условию $\nu\left(A \triangle T_{g} A\right)=0$, выполнено $\nu(A)=0$ или $\nu(A)=1$. 
ПРЕДЛОЖЕНИЕ 15. Мера $\nu \in \mathfrak{M}(\mathfrak{T}, \rho)$ является слабо неразложимой тогда и только тогда, когда она сильно неразложима.

Нам удобно доказывать следующее эквивалентное утверждение.

ПрЕДЛОЖЕНИЕ 16. Пусть $\rho$ - положителъный мультипликативный слабо измеримый кочикл над слабо измеримым действием группы $G$ на стандартном борелевском пространстве $(X, \mathscr{B})$. Пусть $\nu_{1}, \nu_{2} \in \mathfrak{M}(\mathfrak{T}, \rho)$ слабо неразложимые. Тогда либо $\nu_{1}=\nu_{2}$, либо $\nu_{1} \perp \nu_{2}$.

ДоказАТЕЛьство. Пусть $\nu_{1}, \nu_{2} \in \mathfrak{M}(\mathfrak{T}, \rho)$ слабо неразложимы. Рассмотрим разложение Жордана $\nu_{1}$ относительно $\nu_{2}$ и запишем

$$
\nu_{1}=\widetilde{\nu}_{2}+\nu_{3}, \quad \widetilde{\nu}_{2} \ll \nu_{2}, \quad \nu_{3} \perp \nu_{2} .
$$

Поскольку $\nu_{2} \circ T_{g} \ll \nu_{2}$, выполнено также условие $\nu_{2}\left(A \backslash T_{g} A\right)=0$ для каждого $g \in G$. Из этого следует, что для каждого $g \in G$ выполнено $\nu_{1}\left(A \triangle T_{g} A\right)=0$, откуда либо $\nu_{1}(A)=0$, либо $\nu_{1}(A)=1$. Если $\nu_{1}(A)=0$, то $\nu_{1} \perp \nu_{2}$ и утверждение доказано. Если $\nu_{1}(A)=1$, то $\nu_{3}=0$ и $\nu_{1} \ll \nu_{2}$. Положим

$$
\varphi=\frac{d \nu_{1}}{d \nu_{2}}
$$

Так как $\nu_{1}, \nu_{2} \in \mathfrak{M}(\mathfrak{T}, \rho)$ и $\nu_{1} \ll \nu_{2}$, для каждого $g \in G$ функция $\varphi$ удовлетворяет равенству

$$
\varphi\left(T_{g} x\right)=\varphi(x) \quad \nu_{2} \text {-почти наверное. }
$$

Но тогда из слабой неразложимости $\nu_{2}$ следует, что $\varphi=1 \nu_{2}$-почти наверное, и поэтому $\nu_{1}=\nu_{2}$. Предложение доказано.

\section{Список литературы}

[1] С. В. Фомин, "О мерах, инвариантных относительно некоторой группы преобразований”, Изв. АН СССР. Сер. матем., 14:3 (1950), 261-274.

[2] A.I. Bufetov, Finiteness of ergodic unitarily invariant measures on spaces of infinite matrices, 2011, 14 pp., arXiv: 1108.2737.

[3] A. I. Bufetov, "Infinite determinantal measures", Electron. Res. Announc. Math. Sci., 20 (2013), 12-30.

[4] D. Pickrell, "Measures on infinite-dimensional Grassmann manifolds", J. Funct. Anal., 70:2 (1987), 323-356.

[5] A. Borodin, G. Olshanski, "Infinite random matrices and ergodic measures", Comm. Math. Phys., 223:1 (2001), 87-123.

[6] J. von Neumann, "Zur Operatorenmethode in der klassischen Mechanik", Ann. of Math. (2), 33:3 (1932), 587-642.

[7] N. Kryloff, N. Bogoliouboff, "La théorie générale de la mesure dans son application à l'étude des systémes dynamiques de la mécanique non linéaire", Ann. of Math. (2), 38:1 (1937), 65-113.

[8] М. М. Боголюбов, "Про деякі ергодичні властивості суцільних груп перетворень", Наук. зап. Киів. держ. унів. ім. Т. Г. Шевченка. Фіз.-мат. зб., 4:5 (1939), 45-52; Н. Н. Боголюбов, "О некоторых эргодических свойствах непрерывных групп преобразований", Избранные труды, т. 1, Наук. думка, Киев, 1969, 561-569. 
[9] В. А. Рохлин, "О разложении динамической системы на транзитивные компоненты”, Матем. сб., 25(67):2 (1949), 235-249.

[10] В. А. Рохлин, "Об основных понятиях теории меры”, Матем. сб., 25(67):1 (1949), 107-150.

[11] Ю. И. Кифер, С. А. Пирогов, "О разложении квазиинвариантных мер на эргодические компоненты", УМН, 27:5(167) (1972), 239-240.

[12] V.S. Varadarajan, "Groups of automorphisms of Borel spaces", Trans. Amer. Math. Soc., 109:2 (1963), 191-220.

[13] G. Greschonig, K. Schmidt, "Ergodic decomposition of quasi-invariant probability measures", Colloq. Math., 84/85, part 2 (2000), 495-514.

[14] R. R. Phelps, Lectures on Choquet's theorem, 2nd ed., Lecture Notes in Math., 1757, Springer-Verlag, Berlin, 2001, viii+124 pp.

[15] А. М. Вершик, "Описание инвариантных мер для действия некоторых бесконечномерных групп", Докл. АН ССCP, 218:4 (1974), 749-752; англ. пер.: А. M. Veršik, "A description of invariant measures for actions of certain infinite-dimensional groups", Soviet Math. Dokl., 15:5 (1974), 1396-1400.

[16] Г.И. Ольшанский, Унитарные представления бесконечномерных классических грynn, Дис. ... докт. физ.-матем. наук, Ин-т географии АН СССР, М., 1989, $271 \mathrm{c}$.

[17] K. Schmidt, Cocycles on ergodic transformation groups, Macmillan Lectures in Math., 1, Macmillan Company of India, Ltd., Delhi, 1977, 202 pp.

[18] J. Neveu, Martingales à temps discret [Discrete-parameter martingales], Masson et Cie, éditeurs, Paris, 1972, vii+218 pp.

[19] M. Ya. Souslin, "Sur une définition des ensembles mesurables B sans normbres transfinis", C. R. Acad. Sci. Paris, 164:2 (1917), 88-91.

[20] V.I. Bogachev, Measure theory, v. I, II, Springer-Verlag, Berlin, 2007, xviii+500 pp., xiv+575 рр.; пер. с рус.: В.И. Богачев, Основы теории меры, т. 1, 2, НИЦ "Регулярная и хаотическая динамика", М.-Ижевск, 2003, 544 с., 576 с.

[21] Y. Coudène, Théorie ergodique et systèmes dynamiques, notes du cours de DEA, Savoirs Actuels, EDP Sciences/CNRS-Editions, Les Ulis, France, 2013, 196 pp.

\section{Александр Игоревич Буфетов}

(Aleksandr I. Bufetov)

Математический институт им. В. А. Стеклова РАН;

Институт проблем передачи информации РАН

им. А. А. Харкевича, г. Москва;

Национальный исследовательский университет

"Высшая школа экономики", г. Москва;

Aix-Marseille Université, Paris, France;

Rice University, Houston, TX, USA

E-mail: bufetov@mi.ras.ru
Поступила в редакцию 21.12.2012 и 26.08.2013 